\title{
Birational geometry of Fano hypersurfaces of index two
}

\author{
Aleksandr V. Pukhlikov ${ }^{1}$
}

Received: 19 November 2013 / Revised: 25 May 2015 / Published online: 26 December 2015

(C) The Author(s) 2015. This article is published with open access at Springerlink.com

\begin{abstract}
We prove that every non-trivial structure of a rationally connected fibre space on a generic (in the sense of Zariski topology) hypersurface $V$ of degree $M$ in the $(M+1)$-dimensional projective space for $M \geq 16$ is given by a pencil of hyperplane sections. In particular, the variety $V$ is non-rational and its group of birational selfmaps coincides with the group of biregular automorphisms and for that reason is trivial. The proof is based on the techniques of the method of maximal singularities and inversion of adjunction.
\end{abstract}

\section{Introduction}

\subsection{Statement of the main result}

Fix an integer $M \geq 4$. Denote by the symbol $\mathbb{P}$ the complex projective space $\mathbb{P}^{M+1}$. Let $V=V_{M} \subset \mathbb{P}$ be a non-singular hypersurface of degree $M$. Obviously, $V$ is a Fano variety of index two:

$$
\operatorname{Pic} V=\mathbb{Z} H, \quad K_{V}=-2 H,
$$

where $H$ is the class of a hyperplane section. On the variety $V$ there are the following structures of a non-trivial rationally connected fibre space: let $P \subset \mathbb{P}$ be an arbitrary subspace of codimension two, $\alpha_{P}: \mathbb{P} \rightarrow \mathbb{P}^{1}$ the corresponding linear projection, then its restriction

Aleksandr V. Pukhlikov

pukh@liverpool.ac.uk

1 Department of Mathematical Sciences, The University of Liverpool, Liverpool, UK 


$$
\pi_{P}=\left.\alpha_{P}\right|_{V}: V \rightarrow \mathbb{P}^{1}
$$

fibres $V$ into Fano hypersurfaces of index one and for that reason defines on $V$ a structure of rationally connected fibre space. Recall [24], that a (non-trivial) rationally connected fibre space is a surjective morphism $\lambda: Y \rightarrow S$ of projective varieties, where $\operatorname{dim} S \geq 1$ and the variety $S$ and the fibre of general position $\lambda^{-1}(s), s \in S$, are rationally connected (and the variety $Y$ itself is automatically rationally connected by the theorem of Graber, Harris and Starr [9]).

Here is the main result of the present paper.

Theorem 1.1 Assume that $M \geq 16$ and the hypersurface $V$ is sufficiently general (in the sense of Zariski topology on the space of coefficients of homogeneous polynomials of degree $M$ on $\mathbb{P})$. Let $\chi: V \rightarrow Y$ be a birational map onto the total space of a rationally connected fibre space $\lambda: Y \rightarrow S$. Then $S=\mathbb{P}^{1}$ and for some isomorphism $\beta: \mathbb{P}^{1} \rightarrow S$ and some subspace $P \subset \mathbb{P}$ of codimension two we have

$$
\lambda \circ \chi=\beta \circ \pi_{P}
$$

that is, the following diagram commutes:

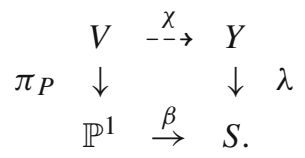

Corollary 1.1 For a generic hypersurface $V$ of dimension $\operatorname{dim} V \geq 16$ the following claims hold.

(i) On the variety $V$ there are no structures of a rationally connected fibre space with the base of dimension $\geq 2$. In particular, on $V$ there are no structures of a conic bundle and del Pezzo fibration, and the variety $V$ itself is non-rational.

(ii) Assume that there is a birational map $\chi: V \rightarrow Y$, where $Y$ is a Fano variety of index $r \geq 2$ with factorial terminal singularities, such that $\mathrm{Pic} Y=\mathbb{Z} H_{Y}$, where $K_{Y}=-r H_{Y}$, and the linear system $\left|H_{Y}\right|$ is non-empty and free. Then $r=2$ and the map $\chi$ is a biregular isomorphism.

(iii) The group of birational self-maps of the variety $V$ coincides with the group of biregular automorphisms:

$$
\text { Bir } V=\text { Aut } V
$$

and for that reason is trivial.

Proof of the corollary. The claims (i-iii) follow from Theorem 1.1 in an obvious way. Q.E.D.

Conjecture 1.1 Assume that $V_{d} \subset \mathbb{P}$ is a smooth hypersurface of degree $d \leq M$, where $d \geq[(M+5) / 2]$ (in that case $V_{d}$ is a Fano variety of index $r=M+2-d$ ). 
Let $\chi: V \rightarrow Y$ be a birational map onto the total space of a rationally connected fibre space $\lambda: Y \rightarrow S$. Then $\operatorname{dim} S \leq r-1$ and if $\operatorname{dim} S=r-1$, then there is a linear subspace $P \subset \mathbb{P}$ of codimension $r$ and a birational map $\beta: \mathbb{P}^{r-1} \rightarrow S$ such that $\lambda \circ \chi=\beta \circ \pi_{P}$, that is, the following diagram commutes

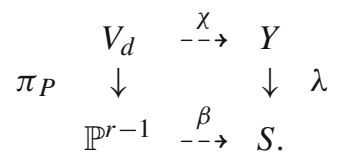

Remark 1.1 For $d \leq M-1$ (that is, for $r \geq 3$ ) one can certainly not expect that all structures of a rationally connected fibre space (or of a Fano-Mori fibre space) are linear projections. Already for a hypersurface of index 3 every pencil of quadrics defines a rational map onto $\mathbb{P}^{1}$, the fibre of which is a complete intersection of the type $2 \cdot(M-1)$ in $\mathbb{P}^{M+1}$, that is, a Fano variety of index one. Conjectures, similar to Conjecture 1.1 have been discussed informally for some time, but until very recently never published, as there were no methods to approach them realistically. The situation is changing now-see the last section in [7].

The purpose of the present paper is to prove Theorem 1.1. As usual, its claim will be derived from a lot more technical and less visual description of maximal singularities of mobile linear systems on $V$. However, before explaining the structure of the proof of Theorem 1.1, let us give a precise meaning to the assumption of the hypersurface $V$ being generic in the sense of Zariski topology.

\subsection{The regularity conditions}

Let

$$
\mathcal{F}=\mathbb{P}\left(H^{0}\left(\mathbb{P}, \mathcal{O}_{\mathbb{P}}(M)\right)\right)
$$

be the space parametrizing hypersurfaces of degree $M$ in $\mathbb{P}$. The local regularity conditions, given below, define an open subset $\mathcal{F}_{\text {reg }} \subset \mathcal{F}$. A separate (but not difficult) problem is to show that for $M \geq 14$ the set $\mathcal{F}_{\text {reg }}$ is non-empty.

Let $o \in \mathbb{P}$ be an arbitrary point, $\left(z_{1}, \ldots, z_{M+1}\right)=\left(z_{*}\right)$ a system of affine coordinates with the origin at the point $o$ and $V \ni o$ a non-singular hypersurface of degree $M$. It is given by an equation $f=0$, where

$$
f=q_{1}+q_{2}+\cdots+q_{M}
$$

is a non-homogeneous polynomial in the variables $z_{*}, q_{i}$ is its homogeneous component of degree $i$. Let $\Pi \subset \mathbb{C}^{M+1}$ be an arbitrary linear subspace of codimension $c \in\{0,1,2,3\}$, on which $q_{1}$ does not vanish identically, that is, $\Pi \not \subset T_{o} V$. We will need the following regularity conditions.

(R1) For any subspace $\Pi$ the sequence of polynomials

$$
\left.q_{1}\right|_{\Pi},\left.q_{2}\right|_{\Pi}, \ldots,\left.q_{M-c}\right|_{\Pi}
$$




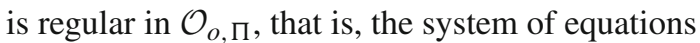

$$
\left.q_{1}\right|_{\Pi}=\left.q_{2}\right|_{\Pi}=\cdots=\left.q_{M-c}\right|_{\Pi}=0
$$

determines a finite set of lines.

(R2) The rank of the quadratic form

$$
\left.q_{2}\right|_{\left\{q_{1}=0\right\}}
$$

is at least $M-\left[\frac{1}{2}(\sqrt{8 M+1}-1)\right]$ (where the square brackets [, ] mean the integral part of a real number).

(R3) The restriction of the equation $q_{3}=0$ onto the quadric hypersurface $\left\{\left.q_{2}\right|_{\Lambda}=0\right\}$, where $\Lambda$ is an arbitrary linear subspace of codimension two in the tangent hyperplane, defines an irreducible reduced closed set.

The last (forth) regularity condition is a global one.

(R4) The intersection of the hypersurface $V$ with an arbitrary linear subspace $P \subset \mathbb{P}$ of codimension two has at most isolated quadratic singularities.

The following claim is true.

Theorem 1.2 For $M \geq 16$ there exists a non-empty Zariski open subset $\mathcal{F}_{\text {reg }} \subset \mathcal{F}$, such that every hypersurface $V \in \mathcal{F}_{\text {reg }}$ is non-singular and satisfies the conditions (R1-R3) at every point, and also the condition (R4).

For the proof of Theorem 1.2 see Sect. 2.6.

\subsection{Plan of the proof of Theorem 1.1}

For an arbitrary subspace $P \subset \mathbb{P}$ of codimension two denote by the symbol $V_{P}$ the blow up of $V$ along the subvariety $V \cap P$. For a mobile linear system $\Sigma$ on $V$ its strict transform on $V_{P}$ denote by the symbol $\Sigma_{P}$. Considering instead of $\Sigma$ its symmetric square, we can always assume that $\Sigma \subset|2 n H|$. Recall that $c_{\text {virt }}(\Sigma)$ is the virtual threshold of canonical adjunction [24, Sec. 2.1]. Theorem 1.1 is an easy corollary from the technical fact formulated below.

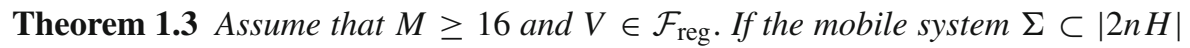
satisfies the inequality

$$
c_{\text {virt }}(\Sigma)<n
$$

then there exists a unique linear subspace $P \subset \mathbb{P}$ of codimension two, such that the subvariety $B=P \cap V$ satisfies the inequality

$$
\operatorname{mult}_{B} \Sigma>n
$$

whereas for the strict transform $\Sigma_{P}$ the following equality holds:

$$
c_{\mathrm{virt}}(\Sigma)=c_{\mathrm{virt}}\left(\Sigma_{P}\right)=c\left(V_{P}, \Sigma_{P}\right) .
$$


The integer $M \geq 16$, the system $\Sigma$ and the integer $n \geq 1$ are fixed throughout the paper. In its turn, Theorem 1.3 will be derived from the following two key facts.

Theorem 1.4 Assume that for some subvariety $B \subset V$ of codimension two the inequality (2) holds. Then $B=P \cap V$, where $P \subset \mathbb{P}$ is a linear subspace of codimension two.

Theorem 1.5 Assume that the inequality (1) holds. Then for some irreducible subvariety $B$ of codimension two the inequality (2) holds.

Theorems 1.4 and 1.5 are given in the order in which they are shown. Theorem 1.5 (the exclusion of the infinitely near case) is the most difficult to prove. Further work is organized as follows.

In Sect. 1, assuming Theorem 1.3, we show Theorem 1.1, and after that, obtain Theorem 1.3, assuming Theorems 1.4 and 1.5. In Sect. 2 we show Theorem 1.4. In Sects. 3-5 we prove Theorem 1.5.

\subsection{Historical remarks and acknowledgements}

The result, completely similar to Theorem 1.1, has been shown for Fano double spaces of index two in [23], see also Chapter 8 in [24]. Prior to the paper [23], the only result giving a complete description of the structures of a rationally connected fibre space on a Fano variety of index two, was Grinenko's theorem $[10,11]$ on the Veronese double cone, a very special Fano threefold.

A series of important results on birational geometry of Fano varieties of index two and higher was obtained by other methods: by the transcendent method of Clemens and Griffiths [3] and its subsequent generalizations (see [2]), and also by means of Kollár's technique $[15,16]$. For the details, see the introduction to the paper [23], where, in particular, the dramatic story of studying the birational geometry of the Veronese double cone and (not completed to this day) studying of the double space of index two is described.

Note that the problem of description of the birational type of Fano varieties of index higher than one was discussed already in the classical paper [13]; Fano himself also worked on the problem (for the cubic threefold $V_{3} \subset \mathbb{P}^{4}$ ) [6].

We would also like to draw the reader's attention to the note [25], where the problem of stable rationality was negatively solved for a large class of Fano hypersurfaces of higher index.

The author thanks the referee for numerous suggestions about improving the exposition and, especially, for spotting a miscalculation in the dimension count that proves that a general hypersurface satisfies the regularity condition (R2).

The results presented in this paper and the techniques used in it were discussed by the author in his talks given in 2009-2014 in Steklov Mathematical Institute. The author is grateful to the members of the divisions of Algebraic Geometry and Algebra and Number Theory for the interest in his work. The author also thanks his colleagues in the Algebraic Geometry research group at the University of Liverpool for the creative atmosphere and general support. 


\section{Pencils of hyperplane sections}

In this section, we prove Theorem 1.1, assuming the claim of Theorem 1.3. After that, Theorem 1.3 is obtained from Theorems 1.4 and 1.5. Finally, we discuss the (routine) proof of Theorem 1.2.

\subsection{Fano fibre spaces over $\mathbb{P}^{1}$}

Let us prove Theorem 1.1. Let $\Sigma \subset|2 n H|$ be the strict transform on $V$ of a free linear system on $Y$, which is the $\lambda$-pull back of a very ample linear system on the base $S$. Then the inequality (1) holds, because $c_{\text {virt }}(\Sigma)=0$. The system $\Sigma \subset|2 n H|$ is now fixed. Assuming the claim of Theorem 1.3, consider the subspace $P \subset \mathbb{P}$ of codimension two, such that for $B=P \cap V$ the inequality (2) holds. Let $\varphi: V^{+} \rightarrow V$ be the blow up of the subvariety $B$ and $E_{B}=\varphi^{-1}(B) \subset V^{+}$the exceptional divisor.

Lemma 2.1 (i) The variety $V^{+}$is factorial and has at most finitely many isolated double points (not necessarily non-degenerate).

(ii) The linear projection $\pi_{\mathbb{P}}: \mathbb{P} \rightarrow \mathbb{P}^{1}$ from the subspace $P$ generates the regular projection

$$
\pi=\pi_{\mathbb{P}} \circ \varphi: V^{+} \rightarrow \mathbb{P}^{1},
$$

the generic fibre of which $F_{t}=\pi^{-1}(t), t \in \mathbb{P}^{1}$ is a non-singular Fano variety of index one, and a finite number of singular fibres have isolated double points.

(iii) The following equalities hold:

$$
\operatorname{Pic} V^{+}=\mathbb{Z} H \oplus \mathbb{Z} E_{B}=\mathbb{Z} K^{+} \oplus \mathbb{Z} F
$$

where $H=\varphi^{*} H$ for simplicity of notations, $K^{+}=K_{V^{+}}$is the canonical class of the variety $V^{+}, F$ is the class of the fibre of the projection $\pi$, where

$$
K^{+}=-2 H+E, \quad F=H-E .
$$

Proof These claims are obvious by the regularity condition (R4) and the well known factoriality of an isolated hypersurface singularity in the dimension 4 and higher, see [1]. (Note that the smoothness of $V$ is contained in the regularity condition (R1).) Indeed, it follows from (R4) that the section of $V$ by a generic hyperplane, containing the subspace $P$, in non-singular, whereas it is well known that any hyperplane section of a non-singular hypersurface in a projective space can have at most isolated singularities. As the fibres of $\pi$ are isomorphic to the sections of $V$ by hyperplanes, containing the subspace $P$, we conclude that $V^{+}$has at most finitely many isolated singular points, hence $V^{+}$is factorial by Grothendieck's theorem [1]. Those isolated singularities are double points as they are double points on the corresponding fibres of $\pi$ by the condition (R1). The rest is trivial. Q.E.D. for the lemma.

Let $\Sigma^{+}$be the strict transform of the system $\Sigma$ on $V^{+}$. 
Lemma 2.2 The linear system $\Sigma^{+}$is composed from the pencil $|F|: \Sigma^{+} \subset|2 n F|$.

Proof For some $m \in \mathbb{Z}_{+}$and $l \in \mathbb{Z}$ we have:

$$
\Sigma^{+} \subset\left|-m K^{+}+l F\right|
$$

where $m=2 n-$ mult $_{B} \Sigma$ and $l=2\left(\operatorname{mult}_{B} \Sigma-n\right) \geq 2$. Thus the threshold of canonical adjunction is

$$
c\left(\Sigma^{+}, V^{+}\right)=m \text {. }
$$

By Theorem 1.3, $c\left(\Sigma^{+}, V^{+}\right)=c_{\text {virt }}(\Sigma)=0$, so that $m=0$ and $l=2 n$, as we claimed. Q.E.D. for the lemma.

Therefore, the mobile linear system $\Sigma$ is composed from the pencil of hyperplane sections, containing $B$, which completes the proof of Theorem 1.1.

\subsection{Mobile systems on the variety $V$}

Assume the claims of Theorems 1.4 and 1.5. Let us prove Theorem 1.3. In the notations of Sect. 1.1 we have to show that for the mobile linear system

$$
\Sigma^{+} \subset\left|-m K^{+}+l F\right|
$$

with $l \in \mathbb{Z}_{+}$the equality

$$
c_{\text {virt }}\left(\Sigma^{+}\right)=c\left(\Sigma^{+}, V^{+}\right)=m
$$

holds. (This is precisely the claim of Theorem 1.3.) Assume the converse:

$$
c_{\text {virt }}(\Sigma)^{+}<m
$$

then the pair $\left(V^{+}, \frac{1}{m} \Sigma^{+}\right)$is not canonical, that is, the linear system $\Sigma^{+}$has a maximal singularity. Since a general fibre of the fibre space $\pi: V^{+} \rightarrow \mathbb{P}^{1}$ is a non-singular birationally rigid variety (in fact, every fibre is birationally superrigid, see, for instance, [5]), the centre of every maximal singularity is contained in some fibre $F_{t}=\pi^{-1}(t)$. Restricting the linear system $\Sigma^{+}$onto such a fibre $F=F_{t}$, we obtain an effective divisor $D \in\left|-m K_{F}\right|$, such that the pair

$$
\left(F, \frac{1}{m} D\right)
$$

is not canonical (in fact, not log canonical, but we do not use that). For any curve $C \subset F, C \cap \operatorname{Sing} F=\emptyset$, it is known, see [24, Chapter 2], that mult ${ }_{C} D \leq m$, which implies that the centre of every non canonical singularity of the pair (3) is either a point, or a curve, passing through a singularity of $F$. Furthermore, it is well known [24, Chapter 7], that a smooth point can not be the centre of a non canonical singularity, 
and the proof of that fact excludes also the case when the centre is a curve (since $F$ has only isolated singularities). Therefore, we may assume that the centre of a maximal (non canonical) singularity of the pair (3) is a singular point $o$.

At this moment, and up to the end of this section, it is convenient to slightly change the notations. We denote the variety $F$ by the symbol $W$. It is a hypersurface of degree $M$ in $\mathbb{P}^{M}$ with an isolated quadratic point $o \in W$. On $W$ there is an effective divisor $D \sim m H$, where $H$ is the class of a hyperplane section of $W$, such that the pair $\left(W, \frac{1}{m} D\right)$ is not canonical at the point $o$. We have to show that this is impossible, that is, to obtain a contradiction. We do it in several steps, modifying the proof in [22].

\subsection{Step 1: Effective divisors on quadrics}

Let $Q \subset \mathbb{P}^{M-1}$ be an irreducible quadric hypersurface of rank $\geq 5, H_{Q} \in \operatorname{Pic} Q=$ $\mathbb{Z} H_{Q}$ the class of a hyperplane section and $B \subset Q$ an irreducible subvariety, which is not contained entirely in Sing $Q$.

Definition 2.1 We say that the effective divisor $D$ on $Q$ satisfies the condition $H(m)$ with respect to $B$, where $m \geq 1$ is a fixed integer, if for any point of general position $p \in B$ (in particular, $p \notin \operatorname{Sing} Q$ ) there exists a hyperplane $F(p) \subset E_{p}$ in the exceptional divisor $E(p)=\varphi_{p}^{-1}(p)$ of the blow up $\varphi_{p}: Q_{p} \rightarrow Q$ of the point $p$, such that the inequality

$$
\operatorname{mult}_{p} D+\operatorname{mult}_{F(p)} \widetilde{D}>2 m
$$

holds, where $\widetilde{D} \subset Q_{P}$ is the strict transform of the divisor $D$.

Note that the divisor $D$ is not assumed to be irreducible, and the integer $m$ does not depend on the point $p$. It is assumed that the hyperplane $F(p)$ depends algebraically on the point $p$. Let $l \geq 1$ be the degree of the hypersurface in $\mathbb{P}^{M-1}$, which cuts out $D$ on $Q$, that is, $D \sim l H_{Q}$.

Now, repeating the proof of Proposition 2.1 in [22] word for word, we obtain

Proposition 2.1 Assume that the inequality

$$
\operatorname{dim} B+\operatorname{rk} Q \geq M+3
$$

holds. Assume, moreover, that an effective divisor $D$ satisfies the condition $H(m)$ with respect to $B$. Then the following alternative takes place:

1. either the inequality $l>2 m$ holds (and we say that this is the simple case),

2. or there is a hyperplane section $Z \subset Q$, which contains entirely the subvariety $B$, such that for a point of general position $p \in B$ in the notations above

$$
F(p)=\widetilde{Z} \cap E_{p},
$$


where $\widetilde{Z} \subset Q_{p}$ is the strict transform of $Z$ on $Q_{p}$, and moreover, $Z$ is contained in the divisor $D$ with the multiplicity

$$
a>2 m-l
$$

(in other words, $D=a Z+D^{*}$, where the effective divisor $D^{*}$ does not contain $Z$ as a component; this case we say to be the hard one).

Remark 2.1 If the quadric $E$ is non-degenerate, that is, $\operatorname{rk} E=M$, then we obtain precisely Proposition 2.1 in [22]. Proof of the latter proposition works in our case without modifications.

\subsection{Step 2: The germ of a quadratic singularity}

In this subsection we consider $o \in W$ as a germ of a quadratic singularity

$$
q_{2}\left(z_{*}\right)+q_{3}\left(z_{*}\right)+\cdots=0
$$

where $\left(z_{*}\right)=\left(z_{1}, \ldots, z_{M}\right)$ (that is, disregarding the embedding $\left.W \subset \mathbb{P}^{M}\right)$, so that $\operatorname{dim} W=M-1$. Let $\varphi: W^{+} \rightarrow W$ be the blow up of the point $o$ and $E=\varphi^{-1}(o) \subset$ $W^{+}$the exceptional divisor, a quadric of rank rk $q_{2}$ in $\mathbb{P}^{M-1}$. Consider an effective divisor $D \ni o$ and assume that for the pair $\left(W, \frac{1}{m} D\right)$ the point $o$ is an isolated centre of a non-canonical singularity. Let $D^{+} \subset W^{+}$be the strict transform of the divisor $D$, so that $D^{+}=\varphi^{*} D-l E$ for some $l \geq 1$. Assume that $l \leq 2 m$, so that the pair $\left(W^{+}, \frac{1}{m} D^{+}\right)$is not $\log$ canonical. Finally, let $S \subset E$ be the centre of a non $\log$ canonical singularity of that pair, which has the maximal dimension, in particular, $S$ is not strictly contained in the centre of another non log canonical singularity, if they exist. Obviously, the inequality

$$
\operatorname{mult}_{S} D^{+}>m
$$

holds.

The following claim generalizes Proposition 2.2 in [22]:

Proposition 2.2 Assume that the inequality

$$
\operatorname{dim} S+\operatorname{rk} q_{2} \geq M+3
$$

holds. Then one of the two cases takes place:

1. either $S$ is a hyperplane section of the quadric $E$ (the simple case),

2. or there exists a hyperplane section $Z \supset S$ of the quadric $E$, satisfying the inequality

$$
\operatorname{mult}_{Z} D^{+}>\frac{2 m-l}{3}
$$


Proof is obtained partially by repeating the proof of Proposition 2.2 in [22] word for word, partially by reduction to that proposition via restricting the divisor $D$ onto a generic section of the singularity $o \in W$ by a linear subspace of dimension rk $q_{2}$.

More precisely, arguing as in [22], we obtain from the inequality (4), that if $S \subset E$ is a prime divisor, then $S \sim H_{E}$ is a hyperplane section of the quadric $E$, that is, the case (1) takes place. Therefore, we assume that $\operatorname{codim}(S \subset E) \geq 2$. Now, arguing as in [22] (replacing Proposition 2.1 in that paper by Proposition 2.1), we obtain that there exists a hyperplane section $Z \supset S$, which is uniquely determined by the log pair $\left(W^{+}, \frac{1}{m} D^{+}\right)$, satisfying the description of the case (2) of Proposition 2.1.

Now let us restrict the divisor $D$ onto the section $W_{\Lambda}=W \cap \Lambda$ of the variety $W \subset$ $\mathbb{C}^{M}$ by a generic linear subspace $\Lambda$ of dimension rk $q_{2}$. In this way we obtain the pair $\left(W_{\Lambda}, \frac{1}{m} D_{\Lambda}\right)$ satisfying the assumptions of Proposition 2.2 in [22] (the germ $o \in W_{\Lambda}$ is a germ of a non-degenerate quadratic singularity), the subvariety $S_{\Lambda}=S \cap W_{\Lambda}^{+}$ is the centre of a non $\log$ canonical singularity of the pair $\left(W_{\Lambda}^{+}, \frac{1}{m} D_{\Lambda}^{+}\right)$, which has the maximal dimension, so that the hyperplane section $Z_{\Lambda}=Z \cap W_{\Lambda}^{+}$satisfies the inequality

$$
\text { mult }_{Z_{\Lambda}} D_{\Lambda}^{+}>\frac{2 m-l}{3}
$$

which by genericity of the linear subspace $\Lambda$ implies the required inequality (5). Proposition 2.2 is shown. Q.E.D.

\subsection{Step 3: Exclusion of the maximal singularity}

Let us come back to the hypersurface $W \subset \mathbb{P}^{M}$ of degree $M$ with an isolated quadratic singularity $o \in W$ of rank $\leq 5$. Let $\varphi: W^{+} \rightarrow W$ be its blow up, $E=\varphi^{-1}(o)$ the exceptional quadric. Consider an effective divisor $D \sim m H$, where $H$ is the class of a hyperplane section of $W$, and let $D^{+} \sim m H-v E$ be its strict transform on $W^{+}$.

Proposition 2.3 The inequality $v \leq \frac{3}{2} m$ holds.

Proof Since the inequality to be shown is linear in $D$, without loss of generality we assume that $D$ is a prime divisor. Assume the converse: $v>\frac{3}{2} m$. To begin with, consider the first hypertangent divisor $D_{2}=\left\{\left.q_{2}\right|_{W}=0\right\}$. Since by the regularity condition (R3) $\left.q_{3}\right|_{E} \not \equiv 0$, we have $D_{2}^{+} \sim 2 H-3 E$, which implies that the divisor $\mathrm{D}_{2}$ is reduced.

Lemma 2.3 The divisor $D_{2}$ is irreducible.

Proof Assume the converse. Then $D_{2}=\Delta_{1}+\Delta_{2}$, where $\Delta_{1,2}$ are distinct hyperplane sections. Since the quadric $E$ is irreducible, $\Delta_{i}^{+} \sim H-\alpha_{i} E$, where $\alpha_{i} \in\{0,1\}$, so that we have $D_{2}^{+}=\Delta_{1}^{+}+\Delta_{2}^{+} \sim 2 H-\alpha E$, where $\alpha \in\{0,1,2\}$. The contradiction proves the lemma. Q.E.D. 
Therefore, $D$ and $D_{2}$ are distinct prime divisors, so that the scheme-theoretic intersection $Y=\left(D \circ D_{2}\right)$ is well defined and satisfies the inequality

$$
\frac{\text { mult }_{o}}{\operatorname{deg}} Y \geq \frac{3}{2} \frac{2 v}{m M}>\frac{9}{2 M} .
$$

Now, repeating the proof of Proposition 3.1 in [22] word for word, we obtain a contradiction by means of the method of hypertangent divisors. Note that here and everywhere else in this paper, whenever the method of hypertangent divisors is used, it is based on the regularity condition (R1), which makes it possible to apply the method to the hypersurface $V$ and its sections by linear subspaces of codimension 1, 2 and 3 . Proposition 2.3 is shown. Q.E.D.

Now let us complete the proof of Theorem 1.3. Assume that the point $o$ is an isolated centre of a non-canonical singularity of the pair $\left(W, \frac{1}{m} D\right)$. By linearity of the Noether-Fano inequality we may assume that $D$ is a prime divisor. Since $v \leq \frac{3}{2} m$, the pair $\left(W^{+}, \frac{1}{m} D^{+}\right)$is not $\log$ canonical and a certain irreducible subvariety $S \subset E$ is the centre of a non $\log$ canonical singularity of that pair. We assume that $S$ has the maximal dimension among all centres of non log canonical singularities of the pair $\left(W^{+}, \frac{1}{m} D^{+}\right.$), so that by [22, Proposition 1.1] we have $\operatorname{dim} S \geq M-5$ (this is a well known consequence of the inversion of adjunction) and by the regularity condition (R2) the assumption of Proposition 2.2 is satisfied.

Proposition 2.4 The subvariety $S$ has codimension at least 2 in the exceptional quadric E.

Proof repeats the proof of Proposition 3.2 in [22] word for word. Following the scheme of arguments in Sec. 3.2 in [22], we conclude that the second case of Proposition 2.2 takes place: there is a hyperplane section $Z \supset S$ of the exceptional quadric $E$, satisfying the inequality (5). Let $P \subset \mathbb{P}^{M}$ be the (unique) hyperplane, cutting out $Z$ on $E$, that is, $W_{P}^{+} \cap E=Z$, where $W_{P}=W \cap P$. Obviously, the prime divisors $W_{P}$ and $D$ are distinct, so that the effective cycle $D_{P}=\left(D \circ W_{P}\right)$ of codimension 2 satisfies the inequality

$$
\text { mult }_{o} D_{P} \geq \operatorname{mult}_{o} D+2 \text { mult }_{Z} D^{+}>\frac{4}{3}(l+m)>\frac{8}{3} m \text {. }
$$

Now consider the pair $\left(W_{P}, \frac{1}{m} D_{P}\right)$. Its strict transform $\left(W_{P}^{+}, \frac{1}{m} D_{P}^{+}\right)$is not log canonical. We may assume that the inequality mult $_{o} D_{P} \leq 4 m$ holds, otherwise we obtain a contradiction, repeating the proof of Proposition 2.3 word for word. The subvariety $S$ is contained in the maximal centre $S^{\prime}$ of a non $\log$ canonical singularity of the pair $\left(W_{P}^{+}, \frac{1}{m} D_{P}^{+}\right.$). It is easy to see that $S^{\prime} \subset E_{P}=Z$ (otherwise, $\operatorname{dim} \varphi\left(S^{\prime}\right) \geq 5$, so that, as $\operatorname{dim}$ Sing $W_{P} \leq 1$, there is a curve $C \subset \varphi\left(S^{\prime}\right), C \cap \operatorname{Sing} W_{P}=\emptyset$, satisfying the inequality mult $_{C} D_{P}>m$, which is impossible for $\left.D_{P} \sim m H_{P}\right)$. For simplicity of notations we assume that $S^{\prime}=S$.

Applying Proposition 2.2 once again, we obtain that one of the following two cases takes place: 
1. either $S$ is a hyperplane section of the quadric $E_{P}$,

2. or there is a hyperplane section $Z^{*} \supset S$ of the quadric $E_{P}$, satisfying the inequality

$$
\text { mult }_{Z^{*}} D_{P}^{+}>\frac{2 m-l^{*}}{3}
$$

where $D_{P}^{+} \sim m H_{P}-l^{*} E_{P}$. By the inequality (6), the integer $l^{*}$ satisfies the inequality $l^{*}>\frac{4}{3} m$. Now, repeating the arguments in the beginning of Sec. 3.3 in [22] word for word (using the regularity condition (R3) instead of the condition (R2.2) in [22]), we exclude the case (1).

Now let us consider the hardest case (2). Since we can not use the strong regularity condition (R2.2) that was used in [22], we need to slightly modify the arguments of Sec. 3.3 in that paper; in particular, we have to assume that $M \geq 14$. Let $R \subset P=$ $\mathbb{P}^{M-1}$ be the unique hyperplane, cutting out $Z^{*}$ on the exceptional quadric $E_{P}$, that is, $W_{R}^{+} \cap E_{P}=Z^{*}$, where $W_{R}=W_{P} \cap R$. Since $W_{R} \sim H_{P}-E_{P}$, the pair $\left(W_{P}^{+}, W_{R}\right)$ is canonical. Furthermore, mult $_{o} W_{R}=2<\frac{8}{3}$, so that by linearity of the inequality (6) and linearity of the condition of non $\log$ canonicity of the pair $\left(W_{P}^{+}, \frac{1}{m} D_{P}^{+}\right)$at $S$, we may assume that $D_{P}$ does not contain the hyperplane section $W_{R}$ as a component (in other words, removing that component, we only make the inequality (6) and the $\log$ Noether-Fano inequality stronger). Therefore, we can take the effective cycle $D_{R}=\left(D_{P} \circ W_{R}\right)$ of codimension 2 on $W_{P}$, which satisfies the inequality

$$
\operatorname{mult}_{o} D_{R} \geq \operatorname{mult}_{o} D_{P}+2 \operatorname{mult}_{Z^{*}} D_{P}^{+}>\frac{28}{9} m \text {. }
$$

Since by the regularity condition (R3) the quadric $\left.q_{2}\right|_{R}=0$ is irreducible and $\left.q_{3}\right|_{R \cap\left\{q_{2}=0\right\}} \not \equiv 0$, we may repeat the proof of Lemma 2.3 and conclude that the divisor $\left.D_{2}\right|_{R}$ is irreducible and has the multiplicity precisely 6 at the point $o$. Therefore,

$$
\frac{\text { mult }_{o}}{\operatorname{deg}}\left(\left.D_{2}\right|_{R}\right)=\frac{3}{M}
$$

Let $Y=Y_{3}$ be an irreducible component of the effective cycle $D_{R}$ with the maximal value of the ratio (mult $\left.{ }_{o} / \mathrm{deg}\right)$. We have

$$
\frac{\text { mult }_{o}}{\operatorname{deg}} Y>\frac{28}{9 M}
$$

so that $Y \neq\left. D_{2}\right|_{R}$, that is, $Y \not \subset D_{2}$ and we may take the effective cycle $\left(Y_{3} \circ D_{2}\right)$ of codimension 2 on $W_{R}$ and 4 on $W$, respectively. At least one of its components $Y_{4}$ satisfies the inequality

$$
\frac{\text { mult }_{o}}{\operatorname{deg}} Y_{4}>\frac{3}{2} \cdot \frac{28}{9 M}=\frac{14}{3 M} .
$$


Now let us apply the technique of hypertangent divisors to the variety $W_{R}$ at the point $o$, satisfying the regularity condition. We obtain a sequence of irreducible subvarieties

$$
Y_{4}, Y_{5}, \ldots, Y_{M-2}
$$

$\operatorname{dim} Y_{i}=M-1-i$, where the curve $Y_{M-2}$ satisfies the inequality

$$
\frac{\text { mult }_{o}}{\operatorname{deg}} Y_{M-2}>\frac{14}{3 M} \cdot \frac{5}{4} \cdot \frac{6}{5} \cdots \frac{M-2}{M-3}=\frac{7(M-2)}{6 M} .
$$

This is impossible for $M \geq 14$.

Proof of Theorem 1.3 is complete. Q.E.D.

\subsection{Regular Fano hypersurfaces}

Let us consider Theorem 1.2. We may prove that a generic Fano hypersurface $V$ satisfies the conditions (R1-R4) separately for each of these conditions.

First, we outline the proof for the condition (R1). It is convenient to start with the following general situation.

Let $\mathcal{P}$ be the linear space, consisting of tuples of homogeneous polynomials $\left(p_{1}, \ldots, p_{N}\right)$ of degrees $\operatorname{deg} p_{i}=i+1$ on the projective space $\mathbb{P}^{N}$, where $N \geq 10$. Consider the closed subset

$$
\mathcal{P}_{\text {non-reg }}=\left\{\left(p_{*}\right) \in \mathcal{P} \mid \operatorname{dim}\left\{p_{1}=\cdots=p_{N}=0\right\} \geq 1\right\} .
$$

Proposition 2.5 The following equality holds:

$$
\operatorname{codim}\left(\mathcal{P}_{\text {non-reg }} \subset \mathcal{P}\right)=\frac{N(N+1)}{2}+2
$$

Remark 2.2 The claim can be made more precise: the closed subset $\mathcal{P}_{\text {non-reg }}$ is reducible and only one of its components has the codimension given above, namely, the component, consisting of such tuples $\left(p_{*}\right)$ that the closed subset $\left\{p_{1}=\cdots=p_{N}=0\right\}$ contains a line in $\mathbb{P}^{N}$. The codimensions of the other components of the set $\mathcal{P}_{\text {non-reg }}$ are higher. However, we do not need this more precise claim.

Proof of Proposition 2.5 is obtained by means of the methods of the papers $[17,18]$ (see also [24, Chapter 3]): it is completely similar to the arguments of [17, Section 1], when regularity of the sequence of polynomials $p_{1}, \ldots, p_{N}$ is violated for the first time for the $i$-th polynomial, $i=1, \ldots, N-2$, and to the arguments of [18, Section 3] in the case when regularity is for the first time violated at one of the last two steps, that is, either the set

$$
\left\{p_{1}=\cdots=p_{N-1}=0\right\} \subset \mathbb{P}^{N}
$$


is a curve and the polynomial $p_{N}$ vanishes identically on one of its components, or the set

$$
\left\{p_{1}=\cdots=p_{N-2}=0\right\} \subset \mathbb{P}^{N}
$$

is a surface and the polynomial $p_{N-1}$ vanishes identically on one of its components.

If the regularity fails for the first time for the $i$-th polynomial, where $i \leq N-2$, the computations are identical to [17, Section 1] and quite elementary, so we leave them to the reader.

In the remaining two cases, we give a sketch of the arguments, based on [18, Section 3]. We consider the case when the regularity first fails at the last step, that is, for $p_{N}$, the other case being similar.

Taking into account Remark 2.2 above, we can assume that the set $\left\{p_{1}=\cdots=\right.$ $\left.p_{N-1}=0\right\}$ is a union of irreducible curves, and $p_{N}$ vanishes on one of them, say $C$, where $\operatorname{deg} C \geq 2$. Set $\langle C\rangle=\mathbb{P}^{k}$ to be the linear span of $C, k \in\{1, \ldots, N\}$. If $k=N$, then the condition $\left.p_{N}\right|_{C} \equiv 0$ imposes at least

$$
N(N+1)+1
$$

independent conditions on the coefficients of $p_{N}$ (see [18, Section 3]), which is more than we need, so we can discard this option and assume that $k \in\{1, \ldots, N-1\}$. Now, following [18, Section 3]), let us fix $\langle C\rangle$, a $k$-plane in $\mathbb{P}^{N}$; then there exists a good sequence of polynomials $p_{i_{1}}, \ldots, p_{i_{k-1}}$, for which $C$ is an associated subvariety (we use the terms and facts from [18, Section 3]). Fixing these polynomials, we get the following set of independent conditions for the remaining ones:

$$
\left.p_{j}\right|_{C} \equiv 0, \quad j \notin\left\{i_{1}, \ldots, i_{k-1}\right\}, 1 \leq j \leq N-1
$$

and $\left.p_{N}\right|_{C} \equiv 0$.

The worst estimate for the codimension of the irregular set corresponds to the choice

$$
\left\{i_{1}, \ldots, i_{k-1}\right\}=\{N-k+1, \ldots, N-1\}
$$

and (taking into account the dimension of the Grassmannian of $k$-planes in $\mathbb{P}^{N}$ ) is given by the expression

$$
f(k)=1+k^{2}+\frac{1}{2} k(N+1-k)(N+2-k)
$$

Calculating the derivative $f^{\prime}(t)$, we see that on the interval $[2, N-1]$ the function $f(t)$ is first increasing (until $t=t_{*} \approx \frac{N}{3}$ ), then decreasing. Therefore, its minimum on that interval is equal to

$$
\min \{f(2), f(N-1)\}
$$

and elementary calculations show that both numbers are higher than $\frac{1}{2} N(N+1)+2$. 
The case when the regularity fails first for $p_{N-1}$, is completely similar. Q.E.D. for Proposition 2.5.

Corollary 2.1 A generic (in the Zariski sense) hypersurface $V$ satisfies the condition (R1) for $M \geq 13$.

Proof In the notations of the condition (R1) it is sufficient to consider the worst case $c=3$. Taking into account the dimension of the Grassmannian of subspaces of codimension 3 in $\mathbb{C}^{M+1}$ and the fact that the point $o \in V$ is arbitrary, by Proposition 2.5 we get that the hypersurface $V$ satisfies the condition (R1), if the inequality

$$
\frac{(M-4)(M-3)}{2}+2-3(M-2)-M \geq 1
$$

holds. It is easy to check that the latter inequality is true for $M \geq 13$. Proof of the corollary is complete.

Now let us look at the remaining conditions (R2-R4).

In order to show that a general Fano hypersurface $V$ satisfies (R2), it is sufficient to demonstrate that for an integer

$$
a>\frac{1}{2}(\sqrt{8 M+1}-1)
$$

the condition rk $\left.q_{2}\right|_{\left\{q_{1}=0\right\}} \leq M-a$ imposes on the coefficients of the quadratic form $q_{2}$ (with $q_{1}$ fixed) at least $M+1$ independent conditions. One checks easily that $\frac{1}{2} a(a+1)$ (the number of independent conditions) is indeed higher than $M$, which proves the claim of Theorem 1.2 for the condition (R2).

Let us consider the condition (R3). The tangent hyperplane at the point $o$ is $\left\{q_{1}=\right.$ $0\}=\mathbb{C}^{M}$, so the subspace $\Lambda$ moves in a $2(M-2)$-dimensional family. For a fixed $\Lambda$, the restriction $\left.q_{2}\right|_{\Lambda}$ by the condition (R2) is of rank at least

$$
r_{M}=M-\left[\frac{1}{2}(\sqrt{8 M+1}-1)\right]-4,
$$

which is at least 6 (for $M=14$ ). Projectivizing the set $\left.q_{2}\right|_{\Lambda}=0$, we get a quadric $G$ of rank at least $r_{M} \geq 6$ in $\mathbb{P}(\Lambda) \cong \mathbb{P}^{M-3}$. Now $G$ is a factorial variety with the Picard group generated by the class of a hyperplane section. Therefore, the restriction $\left.q_{3}\right|_{G}=0$ defines a reducible or non-reduced set if and only if this divisor is a sum of a hyperplane section and a section of $G$ by a quadric in $\mathbb{P}(\Lambda)$. As

$$
h^{0}\left(G, \mathcal{O}_{G}(3)\right)=\left(\begin{array}{c}
M \\
3
\end{array}\right)-M-2 \text { and } h^{0}\left(G, \mathcal{O}_{G}(2)\right)=\left(\begin{array}{c}
M-1 \\
2
\end{array}\right)-1
$$

and $h^{0}\left(G, \mathcal{O}_{G}(1)\right)=M-2$, we see that violation of the condition (R3) imposes (taking into account the dimension of the Grassmannian of subspaces $\Lambda$ )

$$
\frac{1}{6}(M-2)\left(M^{2}-4 M-21\right)+2
$$


independent conditions on the coefficients of the cubic form $q_{3}$, which is higher (and much higher) than $M$. Thus the claim of Theorem 1.2 is shown for the condition (R3).

Finally, let us consider the condition (R4). Unlike the previous three conditions, it is a global one. First, we recall that since $V$ is a smooth hypersurface, $\operatorname{dim} \operatorname{Sing} V \cap P \leq 1$ for any linear subspace $P \subset \mathbb{P}$ of codimension 2 . Thus we need to show that the set of non-singular hypersurfaces $V \in \mathcal{F}$ such that for some subspace $P \subset \mathbb{P}$ of codimension 2 the hypersurface $V \cap P$ in $P$ has at least one irreducible curve $C$ of singular points, has a positive codimension in $\mathcal{F}$. For the curve $C$ we have three options:

1. $C$ is a line,

2. $C$ is a plane curve of degree at least 2 in the plane $\langle C\rangle \cong \mathbb{P}^{2}$,

3. the linear span is a $k$-subspace in $P, k \geq 3$.

It is easy to check that the set of hypersurfaces $V$ such that for some $P$ the hypersurface $V \cap P$ is singular along a line, is of codimension $M^{2}-3 M+3$ in $\mathcal{F}$, so the option (1) is excluded for a general $V$. It is not much harder to exclude the option (2). By the condition (R1), we may assume that the plane $\langle C\rangle \not \subset V$ (through every point $o \in V$ there are only finitely many lines on $V$ ). Now the set of hypersurfaces $V$ such that for some 2-plane $\Pi$ the intersection $V \cap \Pi$ contains a double curve $C$ of degree at least 2 is of codimension $5 M-13$ (this particular value corresponds to the case $\operatorname{deg} C=2$; for higher degrees the codimension is much higher). So we may assume that $\langle C\rangle \cong \mathbb{P}^{k}$, $k \geq 3$ (the option (3) takes place).

For a polynomial $F \in H^{0}\left(\mathbb{P}^{N}, \mathcal{O}_{\mathbb{P}^{N}}(m)\right)$ we denote by $\operatorname{Sing}(F)$ the set

$$
\left\{p \in \mathbb{P}^{N} \mid \frac{\partial F}{\partial X_{0}}(p)=\cdots=\frac{\partial F}{\partial X_{N}}(p)=0\right\}
$$

of singular points of the hypersurface $\{F=0\}$.

Lemma 2.4 Assume that $m \geq 3$. For any set of $k$ linearly independent points $P_{1}, \ldots, P_{k} \in \mathbb{P}^{N}$, where $k \leq N+1$, the condition

$$
\left\{P_{1}, \ldots, P_{k}\right\} \subset \operatorname{Sing}(F)
$$

defines a linear subspace of codimension $k(N+1)$ in $H^{0}\left(\mathbb{P}^{N}, \mathcal{O}_{\mathbb{P}^{N}}(m)\right)$.

Proof We may assume that $P_{1}=(1: 0: 0: \cdots: 0), P_{2}=(0: 1: 0: \cdots: 0)$ etc. correspond to the first $k$ vectors of the standard basis of $\mathbb{C}^{N+1}$. Taking into account that $m \geq 3$, the claim of the lemma becomes obvious. Q.E.D.

Now let us consider a pencil $\Lambda$ of hyperplanes in $\mathbb{P}^{N}$ through a linear subspace $\Pi \subset \mathbb{P}^{N}$ of codimension 2 , Bs $\Lambda=\Pi$. Fix an integer $m \geq 3$ and take any $l=m-2$ distinct hyperplanes

$$
H_{1}, \ldots, H_{l} \in \Lambda \text {. }
$$

Further, consider a set of $k l$ distinct points

$$
\left\{P_{i j} \mid i=1, \ldots, l, j=1, \ldots, k\right\} \subset \mathbb{P}^{N},
$$


such that for every $i=1, \ldots, l$ the points $P_{i j} \in H_{i} \backslash \Pi$ and $\left\{P_{i 1}, \ldots, P_{i k}\right\}$ is a set of linearly independent points in $H_{i}$ (so that $k \leq N$ ).

\section{Lemma 2.5 The condition}

$$
\left\{P_{i 1}, \ldots, P_{i k}\right\} \subset \operatorname{Sing}\left(\left.F\right|_{H_{i}}\right), \quad i=1, \ldots, l,
$$

defines a linear subspace of codimension $k l N$ in $H^{0}\left(\mathbb{P}^{N}, \mathcal{O}_{\mathbb{P}^{N}}(m)\right)$.

Proof It is easier to see this claim in the affine setting: $P_{i j} \in \mathbb{C}^{N}$ with affine coordinates $(u, v)=\left(u_{1}, \ldots, u_{N-1}, v\right)$, the hyperplanes $H_{i}$ are given by the equations $v=\lambda_{i}$, where $\lambda_{1}=0$ and $\lambda_{1}, \ldots, \lambda_{l}$ are distinct. Every (non-homogeneous) polynomial $g(u, v)$ can be written in a unique way as

$$
\begin{aligned}
g(u, v) & =g_{m}(u)+\left(v-\lambda_{1}\right) g_{m-1}(u)+\left(v-\lambda_{1}\right)\left(v-\lambda_{2}\right) g_{m-2}(u)+\cdots \\
& =g_{m}(u)+\sum_{i=1}^{m} g_{m-i}(u) \prod_{e=1}^{i}\left(v-\lambda_{e}\right)
\end{aligned}
$$

where $\left\{\lambda_{1}, \ldots, \lambda_{m}\right\}$ is a set of distinct values, extending the given set $\left\{\lambda_{1}, \ldots, \lambda_{l}\right\}$, and $g_{m-i}$ are polynomials in $u=\left(u_{1}, \ldots, u_{N-1}\right)$ of degree $\leq m-i$, uniquely determined by $g(u, v)$. Now the condition $\left\{P_{11}, \ldots, P_{1 k}\right\} \subset \operatorname{Sing}\left(\left.F\right|_{H_{1}}\right)$ is a condition for the polynomial $g_{m}(u)$, which is the affine form of the polynomial $\left.F\right|_{H_{1}}$, so by Lemma 2.4 it defines a linear subspace of codimension $k N$ in the linear space $\left\{g_{m}(u)\right\}$ of polynomials of degree $\leq m$ in $u_{1}, \ldots, u_{N-1}$. Now fix any polynomial $g_{m}^{+}(u)$ in that subspace. The condition $\left\{P_{21}, \ldots, P_{2 k}\right\} \subset$ Sing $\left(\left.F\right|_{H_{2}}\right)$ defines an affine (not linear) subspace of codimension $k N$ in the linear space $\left\{g_{m-1}(u)\right\}$ of polynomials of degree $\leq m-1$ in $u_{1}, \ldots, u_{N-1}$, whose corresponding linear subspace is given by the condition

$$
\left\{P_{21}, \ldots, P_{2 k}\right\} \subset \operatorname{Sing}\left(g_{m-1}\right)
$$

(Once again, when $g_{m}(u)=g_{m}^{+}(u)$ is fixed.) Continuing in this way, for fixed polynomials

$$
g_{m}^{+}(u), \ldots, g_{m-i+1}^{+}(u)
$$

the condition $\left\{P_{i+1,1}, \ldots, P_{i+1, k}\right\} \subset \operatorname{Sing}\left(\left.F\right|_{H_{i+1}}\right)$ defines an affine subspace in the linear space $\left\{g_{m-i}(u)\right\}$ of polynomials of degree $\leq m-i$ in $u_{1}, \ldots, u_{N-1}$, whose underlying linear subspace is given by the condition

$$
\left\{P_{i+1,1}, \ldots, P_{i+1, k}\right\} \subset \operatorname{Sing}\left(g_{m-i}\right)
$$

so is of codimension $k N$. The last polynomial, to which Lemma 2.4 can be applied, is $g_{3}(u)$. Proof of Lemma 2.5 is complete. 
Corollary 2.2 For a fixed pencil $\Lambda$ and hyperplanes $H_{1}, \ldots, H_{l}$ as above, the condition that there exist points $P_{i j} \in H_{i}, i=1, \ldots, l, j=1, \ldots, k$ as described above, such that

$$
\left\{P_{i 1}, \ldots, P_{i k}\right\} \subset \operatorname{Sing}\left(\left.F\right|_{H_{i}}\right), \quad i=1, \ldots, l,
$$

(that is, the set of points depends on the polynomial $F$ ), defines a subset of codimension $k l$ in $H^{0}\left(\mathbb{P}^{N}, \mathcal{O}_{\mathbb{P}^{N}}(m)\right)$.

Proof Indeed, this is an obvious dimension count, as for the hyperplanes $H_{i}$ being fixed, every point $P_{i j}$ varies in a $(N-1)$-dimensional family. Q.E.D.

Now let us prove the condition (R4) in the case (3). Fix the linear subspace $P \subset \mathbb{P}$ of codimension 2 and consider the set $\mathcal{X}_{P}$ of smooth hypersurfaces $V \subset \mathbb{P}$ of degree $M$ such that $\operatorname{Sing}(V \cap P)$ contains a curve $C$ with $\langle C\rangle$ at least 3-dimensional. The degree of $C$ and the number of such curves in the set $\operatorname{Sing}(V \cap P)$ are bounded by a constant depending on $M$. For a pencil $\Lambda$ of hyperplanes in $P$ and distinct hyperplanes $H_{1}, \ldots, H_{M-2} \in \Lambda$ let

$$
\mathcal{X}_{P}\left(H_{1}, \ldots, H_{M-2}\right) \subset \mathcal{X}_{P}
$$

be the subset defined by the condition that $\operatorname{Sing}(V \cap P)$ contains a curve $C$ with the linear span $\langle C\rangle$ at least 3-dimensional and such that for every $i=1, \ldots, M-2$ the intersection $C \cap H_{i}$ contains $\operatorname{dim}\langle C\rangle$ linearly independent points. It is obvious that $\mathcal{X}_{P}\left(H_{1}, \ldots, H_{M-2}\right)$ is Zariski open in $\mathcal{X}_{P}$ (the curve $C$ depends algebraically on $V$ ). By Corollary 2.2 we have

$$
\operatorname{codim}\left(\mathcal{X}_{P}\left(H_{1}, \ldots, H_{M-2}\right) \subset \mathcal{F}\right) \geq 3(M-2),
$$

so codim $\left(\mathcal{X}_{P} \subset \mathcal{F}\right) \geq 3(M-2)$ as well. As $P$ varies in a $2 M$-dimensional family, the dimension count tells us that in the case (3) the codimension is at least $M-6$.

Proof of Theorem 1.2 is complete.

Remark 2.3 More precise arguments, taking into consideration every possible value of the dimension $\operatorname{dim}\langle C\rangle$, lead to a much stronger estimate in the case (3) of the condition (R4), which is quadratic in $M$.

\section{Subvarieties of codimension two}

In this section we prove Theorem 1.4: if $B$ is a maximal subvariety of codimension two for the system $\Sigma$, then $B$ is a section of the hypersurface $V$ by a linear subspace of codimension two. The proof makes use of the cone technique, see [24, Chapter 2]. The main idea of our arguments is to consider two-dimensional cones, swept out by secant lines of the subvariety $B$. 


\subsection{The secant space of the subvariety $B$}

Assume that the inequality (2) holds. We need to show that $B=P \cap V$, where $P \subset \mathbb{P}$ is a linear subspace of codimension two. If $B$ is contained in a hyperplane, $B \subset \Pi$, then the claim of the theorem is almost obvious: the hyperplane section $V_{\Pi}=V \cap \Pi$ is a factorial variety, Pic $V_{\Pi}=\mathbb{Z} H_{\Pi}$, where $H_{\Pi}$ is the class of a hyperplane section, so that $B \sim m H_{\Pi}$ on $V_{\Pi}$ for some $m \geq 1$. The restriction $\Sigma_{\Pi}$ of the linear system $\Sigma$ onto $V_{\Pi}$ is a non-empty system of divisors, $\Sigma_{\Pi} \subset\left|2 n H_{\Pi}\right|$, whereas mult ${ }_{B} \Sigma_{\Pi}>n$, that is, $B$ is a fixed component of the system $\Sigma_{\Pi}$ of multiplicity (mult $\Sigma_{\Pi}$ ). This implies that $m=1$, so that $B \in\left|H_{\Pi}\right|$ is a hyperplane section of the variety $V_{\Pi} \subset \Pi$, which is what we need.

Starting from this moment, we assume that $B$ is not contained in a hyperplane, that is, $\langle B\rangle=\mathbb{P}$. Let us show that the inequality (2) is impossible for the mobile linear system $\Sigma \subset|2 n H|$. In order to do this, we assume that this inequality is true and show that this assumption leads to a contradiction.

Define the secant space

$$
\operatorname{Sec}(B) \subset B \times B \times \mathbb{P}
$$

as the closure of the set

$$
\operatorname{Sec}^{*}(B) \subset\left(B \times B \backslash \Delta_{B}\right) \times \mathbb{P}, \quad \operatorname{Sec}^{*}(B)=\{(p, q, r) \mid r \in[p, q]\},
$$

where $\Delta_{B} \subset B \times B$ is the diagonal, $[p, q]$ is the line, connecting the distinct points $p, q$. Let $\pi_{B}$ and $\pi_{\mathbb{P}}$ be the projections of the irreducible variety $\operatorname{Sec}(B)$ onto $B \times B$ and $\mathbb{P}$, respectively.

Proposition 3.1 The projection $\pi_{\mathbb{P}}$ is surjective.

Proof is given below in Sect. 2.3.

Proposition 3.1 implies that the image of the restriction of the projection $\pi_{\mathbb{P}}$ onto the set $\operatorname{Sec}^{*}(B)$ contains an open subset in $\mathbb{P}$. In the sequel, speaking about a point $x$ of general position in $\mathbb{P}$, we will always mean, in particular, that $x \notin V$, so that the restriction of the projection from the point $x$ onto $V$ is a finite morphism $V \rightarrow \mathbb{P}^{M}$. Let $\operatorname{Sec}(B, x)$ and $\operatorname{Sec}^{*}(B, x)$ be the fibres of the projection $\pi_{\mathbb{P}}$ and its restriction onto $\operatorname{Sec}^{*}(B, x)$ over a point of general position $x \in \mathbb{P}$.

Obviously, $\operatorname{Sec}(B, x)$ can be considered as a closed subset in $B \times B$, invariant with respect to the involution $\tau:(p, q) \mapsto(q, p)$, and $\operatorname{Sec}^{*}(B, x)$ as a closed subset in $B \times B \backslash \Delta_{B}$, where for a sufficiently general point $x \in \mathbb{P}$ )

$$
\operatorname{Sec}(B, x)=\overline{\operatorname{Sec}^{*}(B, x)} \text {. }
$$

We have $\operatorname{dim} \operatorname{Sec}(B)=2 M-3$, so that $\operatorname{dim} \operatorname{Sec}(B, x)=M-4$. Taking the sections of the closed set $\operatorname{Sec}(B, x)$ by generic very ample divisors on $B \times B$, we obtain for every irreducible component of the $\operatorname{set} \operatorname{Sec}(B, x)$ a dense family of curves $\Gamma$, where 
a general curve $\Gamma$ of the family is not $\tau$-invariant and does not meet any fixed closed subset of codimension $\geq 2$ in $\operatorname{Sec}(B)$. Set

$$
C_{+}=\pi_{1}(\Gamma), \quad C_{-}=\pi_{2}(\Gamma),
$$

where $\pi_{1,2}: B \times B \rightarrow B$ are the projections onto the first and second direct factors, respectively. By construction, $C_{-}$is contained in the cone with the vertex $x$ and the base $C^{+}$and the other way round. The properties of that cone (swept out by the lines $[p, q] \ni x$, for $p, q \in \Gamma$ ) and of the curves $C_{ \pm}$can be made more precise.

The following fact is true.

Proposition 3.2 For some positive integers $d_{C}$ and $d_{R}$ there is an algebraic family

$$
\mathcal{A}=\{A=(C, R, x)\}
$$

of triples $(C, R, x)$, where $C$ is an effective 1 -cycle of degree $d_{C}$ on $\mathbb{P}, R$ is an effective 1-cycle of degree $d_{R}$ on $\mathbb{P}$ and $x \in \mathbb{P}$ is a point, satisfying the following conditions:

1. the projection $\pi_{\mathbb{P}}: \mathcal{A} \rightarrow \mathbb{P}$,

$$
\pi_{\mathbb{P}}:(C, R, x) \mapsto x,
$$

is dominant, that is, $\pi_{\mathbb{P}}(\mathcal{A})$ contains a non-empty Zariski open subset,

2. $C=C_{+}+C_{-}$, where $C_{ \pm}$are distinct irreducible curves, $C_{ \pm} \subset B$,

3. for the cone $C(x) \subset \mathbb{P}$ with the vertex $x$ and the base $C_{+}$we have: $C_{ \pm}$are sections of the cone and the equality

$$
(C(x) \circ V)=C_{+}+C_{-}+R=C+R
$$

holds, where the effective 1-cycle $R$ does not contain $C_{ \pm}$as a component,

4. for any point $p \in C_{ \pm}$we have $x \notin T_{p} C_{ \pm}$,

5. if $p \in C_{ \pm}$is a singular point of the curve $C_{ \pm}$, then $x \notin T_{p} V$,

6. for any point of intersection $p \in C_{+} \cap C_{-}$the generator $[p, x]$ of the cone $C(x)$ has at the point $p$ a simple tangency with the hypersurface $V$ :

$$
([p, x] \cdot V)_{p}=2,
$$

that is, $p \notin R$,

7. the components of the curves $R$ sweep out $V$ :

$$
\overline{\bigcup_{A \in \mathcal{A}} R}=V .
$$

Proof, which makes use of the construction, immediately preceding the statement of Proposition 3.2, is given below in Sect. 2.2.

Let us complete the proof of Theorem 1.4. In the notations of Proposition 3.2, consider an arbitrary irreducible component of the residual curve $R$, which we for 
simplicity denote by the same symbol. Let $D \in \Sigma$ be a generic divisor. By the property (7), we may assume that $R \not \subset D$. Since $B \subset D$ and, moreover, mult $_{B} D>n$, the inequality

$$
2 n \operatorname{deg} R=(R \cdot D) \geq \sum_{p \in R \cap B}(R \cdot D)_{p}>n \sum_{p \in R \wedge B} \operatorname{mult}_{\mathrm{p}} R
$$

holds, where the last sum is taken over the usual and infinitely near points of intersection of the curve $R$ and the subvariety $B$ : the set of those points is denoted by the symbol $R \wedge B$ (see [20]).

Since $C_{ \pm} \subset B$ and, by the property (6), the curve $R$ does not contain the points of the intersection $C_{+} \cap C_{-}$, we have

$$
R \wedge B \supset\left(R \wedge C_{+}\right) \sqcup\left(R \wedge C_{-}\right),
$$

$\sqcup$ means a disjoint union. By the property (5), the curve $R$ meets $C_{ \pm}$at non-singular points of those curves. The following lemma is a version of a very well known claim [20].

Lemma 3.1 The following equality holds:

$$
\sum_{p \in R \wedge C_{ \pm}} \operatorname{mult}_{p} R=\operatorname{deg} R
$$

In the last formula we mean any choice of the sign + or - .

Now from the inequality (9), taking into account (10), we obtain: $2 n \mathrm{deg} R>$ $n(\operatorname{deg} R+\operatorname{deg} R)$, which is impossible. Q.E.D. for Theorem 1.4.

Remark 3.1 Repeating the previous arguments word for word, we exclude the possibility of two maximal subvarieties of codimension two for the system $\Sigma$. Therefore, the section $B=V \cap P$ is uniquely determined.

\subsection{Proof of technical facts}

Let us show Lemma 3.1. By genericity of the curve $C=C_{+}+C_{-}$, each of the curves $C_{ \pm}$is a section of the cone $C(x)$. The normalizations $\widetilde{C_{ \pm}}$of these curves are naturally isomorphic. Let $C^{+}(x)$ be the blow up of the vertex of the cone $C(x)$ and

$$
\widetilde{C}(x)=\widetilde{C}_{ \pm} \times{ }_{C_{ \pm}} C^{+}(x)
$$

the non-singular ruled surface over $\widetilde{C}_{ \pm}$, where the smooth curves $\widetilde{C}_{+}$and $\widetilde{C}_{-}$are realized as its sections. Set $\widetilde{R}$ to be the strict transform of $R$ on $\widetilde{C}(x)$. By the properties (4)-(6) at each point $p \in\left(R \cap C_{+}\right) \sqcup\left(R \cap C_{-}\right)$the corresponding curve $C_{+}$or $C_{-}$is non-singular and transversal to the generator of the cone $[p, x]$, so that

$$
\sum_{q \in R \wedge C_{ \pm}(p)} \operatorname{mult}_{q} R=\sum_{q \in \widetilde{R} \wedge \widetilde{C}_{ \pm}(p)} \operatorname{mult}_{q} \widetilde{R}=\left(\widetilde{R} \cdot \widetilde{C}_{ \pm}\right)_{p}
$$


where the subset $R \wedge C_{ \pm}(p) \subset R \wedge C_{ \pm}$consists of the point $p$ and infinitely near points over it, where the point of the surface $\widetilde{\widetilde{C}}(x)$, corresponding to the point $p$ of intersection of the curves $R$ and $C_{+}$(or $C_{-}$), is denoted by the same symbol $p$. Therefore,

$$
\sum_{p \in R \wedge C_{ \pm}} \operatorname{mult}_{p} R=\sum_{p \in \widetilde{R} \cap C_{ \pm}}\left(\widetilde{R} \cdot \widetilde{C}_{ \pm}\right)_{p}=\left(\widetilde{R} \cdot \widetilde{C}_{ \pm}\right)
$$

but the last number is equal to $\operatorname{deg} R$, see [20]. Q.E.D. for the lemma.

Proof of Proposition 3.2 The construction, immediately preceding the statement of Proposition 3.2, gives an algebraic family $\mathcal{A}$, satisfying the property (1) by Proposition 3.1. Let us show that, somewhat shrinking the family $\mathcal{A}$ (that is, taking a Zariski open subset in that family), one can ensure that the remaining properties (2)-(7) hold. Indeed, our construction yields in the general case distinct irreducible curves $C_{ \pm} \neq C_{-}$, so that the property (2) can be assumed. Proof of Proposition 3.1 implies easily that a generic secant line $[p, q]$ of the variety $B$ is not a 3-secant (see Remark 3.2 in Sect. 2.3 below), that is, $C_{ \pm}$are sections of the cone $C(x)$, whereas $C_{ \pm}$come into the 1-cycle $(C(x) \circ V)$ with multiplicity 1 , which gives the property (3).

For any point $p \in B$ we have $B \not \subset T_{p} V$ (since $B$ is not contained in a hyperplane by assumption), and for that reason for a general point $x \in \mathbb{P}$ the direction of the line $[p, x]$ defines a field of directions on a proper closed subset of the $\operatorname{set} \operatorname{Sec}(B, x)$ (consisting of the points $p \in B$, at which $[p, x] \subset T_{p} B$ ) and for that reason for a general curve $\Gamma$ its projections $C_{ \pm}$are nowhere tangent to the lines $[p, x]$ (that is, at no point $p \in C_{ \pm}$), that is, the property (4) is satisfied (one should also take into account that for a general point $p \in B$ the set

$$
\text { Sing } B \cap T_{p} V
$$

has dimension at most $M-4$, since the hypersurface $V$ is non-singular and the linear system $\sum_{i=0}^{M+1} \lambda_{i}\left(\partial F / \partial x_{i}\right)$ defines a finite morphism $\left.\mathbb{P} \rightarrow \mathbb{P}\right)$.

The property (5) again follows from the fact that $B$ is not contained in a hyperplane: obviously,

$$
\pi_{B}(\operatorname{Sing}(\operatorname{Sec}(B))) \subset(\operatorname{Sing} B \times B) \cup(B \times \operatorname{Sing} B),
$$

so that the point $(p, q) \in \Gamma$ is a singularity of that curve (for a general curve $\Gamma$ ) if and only if $p$ or $q$ belongs to $\operatorname{Sing} B$. Since the pairs of points $(p, q) \in B \times B$ such that $p \in \operatorname{Sing} B$ and $q \in T_{p} V$, form a subset of codimension al least 2, a general curve $\Gamma$ does not contain such pairs. This proves the property (5).

Let us consider the property (6). The subset $\pi_{B}^{-1}\left(\Delta_{B}\right)$ is a closed subset of codimension 1 in $\operatorname{Sec}(B)$, which may consist of several irreducible components of different codimensions. A general curve $\Gamma$ does not intersect the components of codimension two, so we are only interested in the divisorial components.

It is easy to see that the closure of the set

$$
\pi_{B}^{-1}\left(\Delta_{B} \backslash \operatorname{Sing} \Delta_{B}\right) \subset \operatorname{Sec}(B)
$$


is a prime Weil divisor on $\operatorname{Sec}(B)$. For a non-singular point $p \in B \backslash \operatorname{Sing} B$ we have

$$
\pi_{B}^{-1}((p, p))=\{(p, p)\} \times T_{p} B
$$

Let $\left(z_{1}, \ldots, z_{M+1}\right)$ be a system of affine coordinates with the origin at the point $p \in \mathbb{P}$ and

$$
f=q_{1}+q_{2}+\cdots+q_{M}
$$

the equation of the hypersurface $V$. By the condition on the rank of the quadratic form $q_{2}$ we have $\left.q_{2}\right|_{T_{p} B} \not \equiv 0$, so that the set of triples $(p, p, x) \in \operatorname{Sec}(B)$ such that

$$
([p, x] \cdot V)_{p} \geq 3
$$

has in $\operatorname{Sec}(B)$ codimension 2, which is what we need. Therefore, it is sufficient to prove the property (6) for singular points $p \in \operatorname{Sing} B$, that is $(p, p) \in \operatorname{Sing} \Delta_{B}$.

Let $Y \subset \operatorname{Sing} B$ be an irreducible subset of codimension $\geq 2$ with respect to $B$. Since obviously for $p \in Y$

$$
\pi_{B}^{-1}((p, p)) \subset\{(p, p)\} \times T_{p} V
$$

and $\left.q_{2}\right|_{T_{p} V} \not \equiv 0$, we obtain once again, that the set of triples $(p, p, x) \in \operatorname{Sec}(B)$, such that the inequality (11) holds, is of codimension at least two in $\operatorname{Sec}(B)$, which is what we need. Therefore, it is sufficient to consider a divisorial component $Q \subset \operatorname{Sing}(B)$, $\operatorname{codim}(Q \subset B)=1$.

Lemma 3.2 There exists a non-empty Zariski open subset $U_{Q} \subset Q$ such that for any point $p \in U_{Q}$ the set

$$
\pi_{\mathbb{P}}\left(\pi_{B}^{-1}((p, p))\right) \subset \mathbb{P}
$$

is a union of finitely many linear subspaces of dimension $M-1$, contained in $T_{p} V$ and containing $T_{p} Q$.

Proof Straightforward local computations. Since $Q$ is a divisorial component of the set of singular points Sing $B$, over a non-empty Zariski open subset $U_{B} \subset B$ with a non-empty intersection $U_{Q}=U_{B} \cap Q$, the resolution of singularities of the variety $B$ is just the normalization $\widetilde{B} \rightarrow B$, so that at every point $p \in U_{Q}$ the variety $B$ admits a simple analytic parametrization and easy local computations give an explicit description of the limit set of secant lines $[q, r]$ when $q \rightarrow p$ and $r \rightarrow p$. 
We will explain briefly how these limit sets are obtained, leaving it to the reader to perform the computations.

As a model example, let us consider the following problem: determine the limit set of all secant lines $[q, r]$ as $q, r \rightarrow o$, where $C \subset \mathbb{P}^{N}, N \geq 3$, is a (possibly reducible but reduced) curve with a singular point $o \in C$, mult $C_{o} \geq 2$. Even if irreducible, the curve $C$ can have several (possibly singular) branches at $o$. We have therefore to consider the limits of secant lines $[q, r]$, as

(i) $q, r \rightarrow o$ along the same singular branch $C_{1}$ of $C$ (if the branch is non-singular, then the limit is obviously the tangent line to the branch, $T_{o} C_{1}$ ),

(ii) $q \rightarrow o$ along a branch $C_{1}, r \rightarrow o$ along a branch $C_{2}$, where $C_{1} \neq C_{2}$ and $T_{o} C_{1} \neq T_{o} C_{2}$ (one or both of these branches may be non-singular),

(iii) the same as (ii), but the tangent lines coincide, $T_{o} C_{1}=T_{o} C_{2}$.

Let us show that in each of the three cases the limit set of secant lines $[q, r]$ sweeps out a union of 2-planes containing the point $o$. In the case (ii) this is obvious: this set is the plane $\left\langle T_{o} C_{1}, T_{o} C_{2}\right\rangle$. Let us consider the case (i).

Making a linear change of coordinates and re-parameterizing the branch $C_{1}$, we may assume that with respect to a system $\left(z_{1}, \ldots, z_{N}\right)$ of affine coordinates, where $o=(0, \ldots, 0)$, the branch $C_{1}$ has the following parameterization at the point $o$ :

$$
\underline{z}(t)=\sum_{i \geq 0} \underline{v}_{i} t^{d_{i}},
$$

where the sum may be finite or infinite, $d_{i}$ are increasing integers with $d_{0}=$ mult $_{o} C_{1} \geq$ $2,\left\langle\underline{v}_{0}\right\rangle=T_{o} C_{1}, \underline{v}_{i}$ is not parallel to $\underline{v}_{0}$ for all $i$ in the sum above and $\operatorname{gcd}\left(d_{0}, d_{1}, \ldots\right)=$ 1 (so that the parameterization can not be factored through $t \mapsto t^{k}, k \geq 2$ ). Now using 1-dimensional families of secant lines $[q(s), r(s)]$, where

$$
q(s)=\underline{z}(s) \text { and } r(s)=\underline{z}\left(\zeta s+a s^{e}\right),
$$

$\zeta^{d_{0}}=1, e \geq 2$ and $a \in \mathbb{C}$ any constant, it is easy to obtain the limit set of secant lines of this branch as a union of 2-planes. Their explicit description is as follows. Set

$$
i_{1}=\min \left\{j \geq 1 \mid d_{j} \text { is not divisible by } d_{0}\right\}
$$

and inductively

$$
i_{k}=\min \left\{j \geq i_{k-1}+1 \mid d_{j} \text { is not divisible by } m_{k}\right\},
$$

where $m_{k}=\operatorname{gcd}\left(d_{0}, d_{i_{1}}, \ldots, d_{i_{k-1}}\right)$. This procedure terminates, giving us a finite set of indices

$$
i_{0}=0<i_{1}<\cdots<i_{l}
$$

with $\operatorname{gcd}\left(d_{0}, d_{i_{1}}, \ldots, d_{i_{l}}\right)=1$. Now choosing an appropriate root of unity $\zeta$ and a suitable value of $e$ in the parameterization above, it is easy to check that the limit set 
of the branch $C_{1}$ is the union of planes

$$
\left\langle\underline{v}_{0}, \underline{v}_{i_{k}}\right\rangle, \quad k=1, \ldots, l .
$$

We leave the computations to the reader as well as checking that for any parameterization of $q(s)$ and $r(s)$ the limit of the secant line $[q(s), r(s)]$ is inside the union of the planes above.

This completes the case (i). The case (iii) is very similar to (i) and is also left to the reader.

Keeping in mind this model example, let us come back to the proof of Lemma 3.2.

At a generic point $p \in Q$ we choose a system of linear coordinates

$$
\left(z_{1}, z_{2}, z_{3}, z_{4}, y_{1}, \ldots, y_{M-3}\right)=(z, y)
$$

with the origin at the point $p$, such that the projection $T_{p} Q \rightarrow\left\{z_{1}=z_{2}=z_{3}=\right.$ $\left.z_{4}=0\right\}$ is an isomorphism, so that near $p$ the non-singular subvariety $Q$ admits a parameterization of the form

$$
(z, y)=\left(\underline{v}_{-1}(y), y\right)
$$

Near $p$ the subvariety $B$ is a union of branches, each of which admits a uniform parameterization

$$
\underline{v}_{-1}(y)+\sum_{i \geq 0} \underline{v}_{i}(y) t^{d_{i}}
$$

satisfying the same properties as the local parameterization of the branch $C_{1}$ discussed above (which can be seen, as we mentioned in the beginning of the proof of this lemma, through considering the normalization $\widetilde{B} \rightarrow B$ ).

Now elementary local computations, similar to those sketched above for the 1dimensional case, show that the limit set of secant lines at the point $p$ is a union of linear space of dimension $M-1$, containing $T_{p} Q$ (and, naturally, contained in $T_{p} V$ ). We leave these easy explicit computations to the reader. Proof of Lemma 3.2 is complete.

Now for any point $p \in U_{Q}$ and some linear subspace $\Pi \subset \pi_{\mathbb{P}}\left(\pi_{B}^{-1}((p, p))\right)$ we have $\left.q_{2}\right|_{\Pi} \not \equiv 0$, so that the closed set

$$
\left\{q_{2}=0\right\} \cap \pi_{\mathbb{P}}\left(\pi_{B}^{-1}((p, p))\right) \subset \mathbb{P}
$$

is of dimension $M-2$. Therefore, the set of triples $(p, p, x) \in \operatorname{Sec}(B)$, satisfying the inequality (11), where $p \in U_{Q}$, has the dimension $(M-3)+(M-2)=2 M-5$, that is, the codimension 2 in $\operatorname{Sec}(B)$. This completes the proof of the property (6).

Finally, the property (7) is obvious (for instance, follows immediately from the proof of Proposition 2.1, given below).

Proposition 3.2 is shown. Q.E.D. 


\subsection{The secant variety}

Set

$$
\operatorname{Sec}(B)=\pi_{\mathbb{P}}(\operatorname{Sec}(B))=\overline{\bigcup_{B \ni p \neq q \in B}[p, q]} \subset \mathbb{P}
$$

and let us call $\operatorname{Sec}(B)$ the secant variety of the subvariety $B \subset \mathbb{P}$ (as opposed to the secant space, introduced in $\operatorname{Sect} .2 .1)$. We need to show that $\operatorname{Sec}(B)=\mathbb{P}$. Let $\alpha: \mathbb{C}^{M+2} \backslash\{0\} \rightarrow \mathbb{P}$ be the canonical projection. For a closed set $Y \subset \mathbb{P}$ the symbol $Y^{\text {aff }}$ stands for the affine cone

$$
\overline{\alpha^{-1}(Y)}=\alpha^{-1}(Y) \cup\{0\} \subset \mathbb{C}^{M+2} .
$$

Let $\sigma: B^{\text {aff }} \times B^{\text {aff }} \times \mathbb{C}^{2} \rightarrow \mathbb{C}^{M+2}$ be the map of taking the linear combination

$$
\sigma:(v, w,(\lambda, \mu)) \mapsto \lambda v+\mu w .
$$

Obviously, $\operatorname{Sec}(B)^{\text {aff }}$ is the closure of the image of the map $\sigma$. Furthermore, it is obvious that for a non-singular point $p \in B$ the tangent space $T_{v} B^{\text {aff }}$ does not depend on the choice of a non-zero vector $v \in \alpha^{-1}(p)$ and for that reason we denote it by the symbol $T_{p} B^{\text {aff }}$. It is clear that the embedded tangent space $T_{p} B \subset \mathbb{P}$ satisfies the equality

$$
\left(T_{p} B\right)^{\text {aff }}=T_{p} B^{\text {aff }} .
$$

Let $p, q \in B$ be a pair of non-singular points. Obviously, the differential $d \sigma$ at the point $\left(v_{0}, w_{0},\left(\lambda_{0}, \mu_{0}\right)\right)$ is

$$
\begin{aligned}
& d \sigma: T_{p} B^{\text {aff }} \times T_{q} B^{\text {aff }} \times \mathbb{C}^{2} \rightarrow \mathbb{C}^{M+2}, \\
& d \sigma:(v, w,(\lambda, \mu)) \mapsto \lambda_{0} v+\mu_{0} w+\lambda v_{0}+\mu w_{0},
\end{aligned}
$$

so that for a non-singular point $r \in \operatorname{Sec}(B), r \in[p, q]$, we have $T_{r} \operatorname{Sec}(B)^{\text {aff }}=$ $\operatorname{Im} d \sigma=T_{p} B^{\text {aff }}+T_{q} B^{\text {aff }}$ (taking into account that $v_{0} \in T_{p} B^{\text {aff }}$ and $w_{0} \in T_{q} B^{\text {aff }}$ ). Set

$$
T(p, q)=T_{p} B^{\mathrm{aff}}+T_{q} B^{\mathrm{aff}}
$$

Therefore, $\operatorname{Sec}(B)=\mathbb{P}$ if and only if $d \sigma$ is surjective, that is $T(p, q)=\mathbb{C}^{M+2}$.

Remark 3.2 At this point we observe that the equality $T(p, q)=\mathbb{C}^{M+2}$ implies that a general secant line is not a 3 -secant.

Assume now that $\operatorname{Sec}(B) \neq \mathbb{P}$ is a proper irreducible subvariety. $\operatorname{Since} \operatorname{codim}(B \subset$ $\mathbb{P})=3$, this implies that

$$
\operatorname{codim}\left(T_{p} B^{\text {aff }} \cap T_{q} B^{\text {aff }}\right) \leq 5,
$$


and the latter holds for any non-singular points $p, q \in B$. Let us show that our assumption leads to a contradiction.

The symbol $\pi_{p}$ stands for the linear projection $\mathbb{P}-\rightarrow \mathbb{P}^{2}$ from the tangent space $T_{p} B$ for a non-singular point $p \in B$. The projection $\pi_{p}$ is the projectivization of the linear map

$$
\pi_{p}^{\mathrm{aff}}: \mathbb{C}^{M+2} \rightarrow\left(\mathbb{C}^{M+2} / T_{p} B^{\mathrm{aff}}\right) \cong \mathbb{C}^{3}
$$

The differential of the restriction of the latter map onto $B^{\text {aff }}$ is not surjective at a point of general position. Indeed, for any smooth point $q \in B$ we have:

$$
\operatorname{dim} \pi_{p}^{\mathrm{aff}}\left(T_{q} B^{\mathrm{aff}}\right) \leq 2
$$

Therefore, $\pi_{p}(B) \neq \mathbb{P}^{2}$ and for that reason $\pi_{p}(B)$ is either a point or some irreducible curve $C \subset \mathbb{P}^{2}$. If $\pi_{p}(B)$ is a point or $C$ is a line, then the subvariety $B$ is contained in a hyperplane, which contradicts the assumption. Therefore, $\pi_{p}(B)=C$ is a curve of degree $d \geq 2$.

Let $c \in C$ be a point of general position,

$$
B_{c}=\overline{\left(B \cap \pi_{p}^{-1}(c)\right) \backslash T_{p} B}
$$

the fibre of the projection $\left.\pi_{p}\right|_{B}$. Obviously, $B_{C}$ is a closed subset of pure codimension two in the fibre $\overline{\pi_{p}^{-1}(c)} \cong \mathbb{P}^{M-1}$. For that reason the secant variety $\operatorname{Sec}\left(B_{c}\right)$ coincides with its linear span $\left\langle B_{c}\right\rangle$ (it is sufficient to check this almost obvious fact for a curve in $\mathbb{P}^{3}$ ).

Therefore, we have three options:

1. $\operatorname{Sec}\left(B_{c}\right)=\mathbb{P}^{M-1}$,

2. $\operatorname{Sec}\left(B_{c}\right)$ is a hyperplane in $\overline{\pi_{p}^{-1}(c)} \cong \mathbb{P}^{M-1}$,

3. $\operatorname{Sec}\left(B_{c}\right)=B_{c}$ is a subspace of codimension two in $\mathbb{P}^{M-1}$.

Assume that the case (1) takes place. Since $\operatorname{Sec}\left(B_{c}\right) \subset \operatorname{Sec}(B)$, we have

$$
\overline{\pi_{p}^{-1}(C)} \subset \operatorname{Sec}(B)
$$

On the left we have an irreducible divisor in $\mathbb{P}$, so that by our assumption that $\operatorname{Sec}(B) \neq \mathbb{P}$ the equality $\operatorname{Sec}(B)=\overline{\pi_{p}^{-1}(C)}$ holds. However, it is obvious, that $\operatorname{Sec}(B)$ contains points outside the set $\overline{\pi_{p}^{-1}(C)}$ : let $c_{1}, c_{2} \in C$ be a general pair of points, $q_{i} \in B_{c_{i}}$ general points, then $\left[q_{1}, q_{2}\right] \subset \operatorname{Sec}(B)$, but $\pi_{p}\left(\left[q_{1}, q_{2}\right]\right)=\left[c_{1}, c_{2}\right] \not \subset C$. This contradiction excludes the case (1).

The case (3) is impossible, as $V$ does not contain linear subspaces of dimension $M-3$.

Therefore, the case (2) takes place. Again we take a general pair of points $c_{1}, c_{2} \in C$. Let $L=\left[c_{1}, c_{2}\right] \subset \mathbb{P}^{2}$ be the line through them, $H=\overline{\pi_{p}^{-1}(L)}$ the corresponding 
hyperplane in $\mathbb{P}$. Set also

$$
P_{i}=\overline{\pi_{p}^{-1}\left(c_{i}\right)} \subset H \cong \mathbb{P}^{M}
$$

The linear space $P=T_{p} B$ is of codimension two in $H$ and $P_{1} \cap P_{2}=P$. Furthermore, set

$$
B_{i}=B_{c_{i}} \text { and } \Pi_{i}=\operatorname{Sec}\left(B_{i}\right)=\left\langle B_{i}\right\rangle
$$

these are hyperplanes in $P_{i}, i=1,2$.

Proposition 3.3 The following equality holds:

$$
\operatorname{Sec}\left(B_{1} \cup B_{2}\right)=H \text {. }
$$

It is clear that since the points $c_{1}, c_{2}$ are general, Proposition 3.3 implies the equality $\operatorname{Sec}(B)=\mathbb{P}$, which contradicts the initial assumption and proves Proposition 3.1.

Proof of Proposition 3.3 None of the irreducible components of the sets $B_{1}, B_{2}$ is a cone, as by the regularity condition (R1) there are only finitely many lines through every point on $V$. Let $\Lambda \subset H$ be a 5-dimensional subspace of general position, $Q_{i}=P_{i} \cap \Lambda$ and $S_{i}=B_{i} \cap \Lambda, i=1,2$. Now $S_{1}, S_{2}$ are (possibly reducible) surfaces in $\Lambda \cong \mathbb{P}^{5}$, the linear spans $\left\langle S_{i}\right\rangle$ of which are 3-planes $R_{i}=\Pi_{i} \cap \Lambda$. The components of the surfaces $S_{1}, S_{2}$ are not cones and for that reason

$$
\bigcap_{s \in S_{i}} T_{S} S_{i}=\emptyset
$$

for $i=1$, 2. If $R_{12}=R_{1} \cap R_{2}$ is a line, then we conclude that for a general pair of points $\left(s_{1}, s_{2}\right) \in S_{1} \times S_{2}$ the planes $T_{S_{1}} S_{1}$ and $T_{S_{2}} S_{2}$ are disjoint. This implies, that $\operatorname{Sec}\left(S_{1} \cup S_{2}\right)=\Lambda$, so that Proposition 3.3 is shown in this case.

Therefore we assume that $R_{12}$ is a plane, that is,

$$
R_{1} \cap R_{2}=R_{1} \cap Q_{2}=Q_{1} \cap R_{2}
$$

By the genericity of the subspace $\Lambda$ this means that

$$
\Pi_{12}=\Pi_{1} \cap \Pi_{2}=\Pi_{1} \cap P_{2}=P_{1} \cap \Pi_{2},
$$

and for that reason $\Pi_{12}=\Pi_{1} \cap P=\Pi_{2} \cap P$. The points $c_{1}, c_{2}$ are chosen independently of each other, so that we can conclude that there exists (a uniquely determined) hyperplane $Q \subset P$ such that for a point of general position $c \in C$ we have

$$
\left\langle B_{c}\right\rangle=\operatorname{Sec}\left(B_{c}\right) \supset Q .
$$

Let $\pi_{Q}: \mathbb{P}-\rightarrow \mathbb{P}^{3}$ be the projection from the linear subspace $Q$. By what we proved, $\pi_{Q}\left(\left\langle B_{c}\right\rangle\right)$ is a point and for that reason $\pi_{Q}\left(B_{C}\right)$ is a point, so that the image $\pi_{Q}(B)$ 
is a curve $C^{+}$(the projection of which from the point $\pi_{Q}(P)$ is the curve $C \subset \mathbb{P}^{2}$ ). If $C^{+}$is contained in some plane in $\mathbb{P}^{3}$, then $B$ is contained in some hyperplane in $\mathbb{P}$, either, which contradicts our assumption. Thus

$$
\left\langle C^{+}\right\rangle=\operatorname{Sec}\left(C^{+}\right)=\mathbb{P}^{3} .
$$

Now let $\xi_{1}, \xi_{2} \in C^{+}$be a general pair of points, $\Lambda_{i}=\pi_{Q}^{-1}\left(\xi_{i}\right) \subset \mathbb{P}$ the corresponding subspaces of codimension $3, B_{i}^{+}=\overline{\pi_{Q}^{-1}\left(\xi_{i}\right) \cap B}$ the fibres of the projection $\left.\pi_{Q}\right|_{B}$. We know that $B_{i}^{+} \subset \Lambda_{i}$ are hypersurfaces (possibly reducible) and

$$
\left\langle B_{i}^{+}\right\rangle=\operatorname{Sec}\left(B_{i}^{+}\right)=\Lambda_{i}
$$

Since $B_{i}^{+}$are not cones, we conclude that

$$
\operatorname{Sec}\left(B_{i}^{+} \cup B_{2}^{+}\right)=\pi_{Q}^{-1}\left(\left[\xi_{1}, \xi_{2}\right]\right),
$$

whence, finally, it follows that $\operatorname{Sec}(B)=\mathbb{P}$. Proof of Propositions 3.3 and 3.1 is complete.

\section{Infinitely near case: I-preparatory work}

In this section we start the proof of Theorem 1.5, that is, the exclusion of the infinitely near case. Here we carry out preparatory work: we come over to a hyperplane section of the hypersurface $V$, in order to use the $8 n^{2}$-inequality, list all particular cases that need to be considered and obtain aprioric estimates for the multiplicity of the selfintersection. We use the following tools: the inversion of adjunction, the technique of counting multiplicities and the method of hypertangent divisors.

\subsection{The method of hypertangent divisors}

Let $\Sigma \subset|2 n H|$ be a mobile linear system with no maximal subvarieties of codimension two. Fix a maximal singularity $E^{*} \subset \widetilde{V}$ of the system $\Sigma$ with the centre $B \subset V$ of maximal dimension.

Lemma 4.1 $B$ is a point or a curve on $V$.

Proof By the $4 n^{2}$-inequality we have

$$
\operatorname{mult}_{B} Z>4 n^{2}
$$

where $Z=\left(D_{1} \circ D_{2}\right)$ is the self-intersection of the system $\Sigma$. Since $Z \sim 4 n^{2} H^{2}$, by [19, Proposition 5] it follows that $\operatorname{dim} B \leq 1$. Q.E.D. for the lemma.

The cases $\operatorname{dim} B=1$ and $\operatorname{dim} B=0$ are dealt with in word for word the same way, the assumption on the existence of a maximal singularity leads to a contradiction, 
excluding both cases. We will assume that $B=o$ is a point (if $B$ is a curve, take a general point $o \in B$, and then the proof for the case $\operatorname{dim} B=0$ applies; note that all local information about the linear system $\Sigma$ at the point $o$ and its strict transform with respect to the blow up of this point is obtained through restricting the system onto the section of $V$ by a generic linear subspace through $o$ in the $\operatorname{dim} B=0$ case, so the arguments work in the $\operatorname{dim} B=1$ case as well). The following fact is true.

Proposition 4.1 The following inequality holds: mult $_{o} \Sigma \leq 3 n$.

Proof Assume the converse: mult $_{o} \Sigma>3 n$. Let $T=T_{o} V \cap V$ be the intersection of the hypersurfaces $V$ with the tangent hyperplane. Obviously,

$$
T \subset T_{o} V \cong \mathbb{P}^{M}
$$

is a Fano hypersurface with the isolated double point $o \in T$. The tangent cone at the point $o$ is the quadric $\left\{\left.q_{2}\right|_{\left\{q_{1}=0\right\}}=0\right\}$. For a generic divisor $D \in \Sigma$ we have $D \neq T$, so that $(D \circ T)$ is an effective cycle of codimension two, satisfying the inequality

$$
\operatorname{mult}_{o}(D \circ T)>6 n \text {. }
$$

Let $Y$ be a component of the cycle $(D \circ T)$ with the maximal value of the ratio (mult ${ }_{o} / \mathrm{deg}$ ). Therefore, the prime divisor $Y \subset T$ satisfies the inequality

$$
\frac{\text { mult }_{o}}{\operatorname{deg}} Y>\frac{3}{M} \text {. }
$$

The first hypertangent divisor $T_{2}=\left\{\left.q_{2}\right|_{T}=0\right\}$ is irreducible and by the regularity conditions mult $T_{o}=6, \operatorname{deg} T_{2}=2 M$, so that $T_{2} \neq Y$. Let us form the effective cycle $\left(\left\{\left.q_{2}\right|_{T}=0\right\} \circ Y\right)$ and choose in it an irreducible component $Y_{3}$ with the maximal value of the ratio (mult ${ }_{o} / \mathrm{deg}$ ). Now we apply to $Y_{3}$ the standard technique of hypertangent linear systems [24, Chapter 3]: take generic hypertangent divisors $T_{i}$, that is, the divisors, the strict transforms $T_{i}^{+}$of which on the blow up $V^{+}$of the point $o$ with the exceptional divisor $E \cong \mathbb{P}^{M-1}$ are

$$
T_{i}^{+} \in|i H-(i+1) E|, \quad i=4, \ldots, M-1,
$$

and construct a sequence of irreducible subvarieties

$$
Y_{3}, Y_{4}, \ldots, Y_{M-1}
$$

where $\operatorname{codim}\left(Y_{i} \subset V\right)=i$, and $Y_{i+1}$ is an irreducible component of the effective cycle $\left(Y_{i} \circ T_{i+1}\right)$ with the maximal value of (mult $t_{o} / \mathrm{deg}$ ). For the curve $Y_{M-1}$ we have the inequality

$$
1 \geq \frac{\text { mult }_{o}}{\operatorname{deg}} Y_{M-1}>\frac{3}{M} \cdot \frac{3}{2} \cdot \frac{5}{4} \cdot \frac{6}{5} \cdots \cdot \frac{M}{M-1}=\frac{9}{8} .
$$

This contradiction proves our proposition. Q.E.D. 
Arguing in a similar way, we obtain the following fact.

Proposition 4.2 For any irreducible subvariety $Y \subset V$ of codimension two the following inequality holds:

$$
\frac{\text { mult }_{o}}{\operatorname{deg}} Y \leq \frac{3}{M}
$$

Proof Set again $T=T_{o} V \cap V$. If $Y \subset T$, that is, $Y$ is a prime divisor on the hypersurface $T \subset T_{o} V \cong \mathbb{P}^{M}$, then we argue in word for word the same way as in the proof of Proposition 4.1, deriving a contradiction from the assumption that the inequality (13) does not hold.

Assume now, that $Y \not \subset T$ and the inequality (13) is not true. Then for the effective cycle $(Y \circ T)$ the inequality

$$
\frac{\text { mult }_{o}}{\operatorname{deg}}(Y \circ T)>\frac{6}{M}
$$

holds, so that there is an irreducible component $Y_{3}$ of the cycle $(Y \circ T)$, satisfying that inequality. Now we argue in the same way as in the proof of Proposition 4.1: taking generic hypertangent divisors $T_{4}, \ldots, T_{M-1}$, we construct a sequence of irreducible subvarieties $Y_{3}, Y_{4}, \ldots, Y_{M-1}$, the last one of which is a curve, satisfying the inequality

$$
1 \geq \frac{\text { mult }_{o}}{\operatorname{deg}} Y_{M-1}>\frac{6}{M} \cdot \frac{5}{4} \cdot \frac{6}{5} \cdots \cdots \frac{M}{M-1}=\frac{6}{4} .
$$

This contradiction completes the proof of the proposition.

Remark 4.1 The second part of the proof of Proposition 3.2 gives a much stronger estimate for the ratio (mult ${ }_{o} / \mathrm{deg}$ ) in the case $Y \not \subset T$ :

$$
\frac{\text { mult }_{o}}{\operatorname{deg}} Y \leq \frac{2}{M}
$$

Now let $\Pi \subset \mathbb{P}$ be a linear subspace of codimension 1 or 2 , containing the point $o$, but not contained in the hyperplane $T_{o} V$, so that $V_{\Pi}=V \cap \Pi$ is an irreducible hypersurface of degree $M$ in $\Pi$, non-singular at the point $o$. Let $Y \subset V_{\Pi}$ be an irreducible subvariety of codimension two.

Proposition 4.3 (i) If $Y \subset T_{o} V_{\Pi}$, then for $\operatorname{codim}(\Pi \subset \mathbb{P})=j \in\{1,2\}$ the following estimate holds:

$$
\frac{\operatorname{mult}_{o}}{\operatorname{deg}} Y \leq \frac{1}{M} \max \left(3, \frac{8 M}{3(M-j)}\right)
$$


(ii) If $Y \not \subset T_{o} V_{\Pi}$, then for $\operatorname{codim}(\Pi \subset \mathbb{P})=j \in\{1,2\}$ the following estimate holds

$$
\frac{\text { mult }_{o}}{\operatorname{deg}} Y \leq \frac{1}{M}\left(\frac{2 M}{M-j}\right)
$$

(The somewhat strange writing of the right hand part of the inequality (ii) will become clear below.)

Proof (i) Repeating the arguments of the first part of the proof of Proposition 4.2 word for word and taking into account the regularity conditions for the hypersurface $V_{\Pi}$, we obtain the inequality

$$
1>\frac{3}{M} \cdot \frac{3}{2} \cdot \frac{5}{4} \cdot \frac{6}{5} \cdots \cdot \frac{M-1}{M-2}=\frac{9}{8}\left(1-\frac{1}{M}\right)
$$

for $\operatorname{codim}(\Pi \subset \mathbb{P})=1$, and the inequality

$$
1>\frac{3}{M} \cdot \frac{3}{2} \cdot \frac{5}{4} \cdot \frac{6}{5} \cdots \cdot \frac{M-2}{M-3}=\frac{9}{8}\left(1-\frac{2}{M}\right)
$$

for $\operatorname{codim}(\Pi \subset \mathbb{P})=2$. It is easy to see that these inequalities are impossible. The contradiction proves the claim (i).

In the case (ii) we repeat the arguments of the second part of the proof of Proposition 4.2 word for word, once again taking into account that by the considerations of dimension we take $\operatorname{codim}(\Pi \subset \mathbb{P})=1$ or 2 hypertangent divisors less. Again we get a contradiction, which proves the claim (ii).

Proof of Proposition 4.3 is complete.

\subsection{The restriction onto a hyperplane section}

The next step in the proof of Theorem 1.5 is the restriction of the linear system $\Sigma$ onto a suitable hyperplane section of the variety $V$, which allows us to make the estimate for the multiplicity of the self-intersection at the point $o$ twice stronger. If the inequality mult $_{o} Z>8 n^{2}$ holds (where $Z$ is the self-intersection of the mobile linear system $\Sigma$ ), then this step can be skipped, considering below instead of the hyperplane section $P \ni o$ the hypersurface $V$ itself: in that case, the dimension does not drop and all estimates become only stronger, so that the proof given below works without any modifications. Keeping this in mind, assume that mult $Z \leq 8 n^{2}$. Recall that $V^{+}$is the blow up of $V$ at the point $o$ with the exceptional divisor $E \cong \mathbb{P}^{M-1}$. The following fact is true.

Proposition 4.4 (The $8 n^{2}$-inequality) There exists a subspace $\Pi \subset$ E of codimension 2 (uniquely determined by the system $\Sigma$ ), satisfying the inequality

$$
\operatorname{mult}_{o} Z+\operatorname{mult}_{\Pi} Z^{+}>8 n^{2} .
$$


Proof This is [23, Proposition 4.1].

Now let us consider the linear system $|H-\Pi|$, consisting of hyperplane sections that cut out $\Pi$ on $E$, that is, for a general divisor $P \in|H-\Pi|$ we have: $P \in|H|$ is a hyperplane section, smooth at the point $o$ and $P^{+} \supset \Pi$. Obviously,

$$
\operatorname{dim}|H-\Pi|=2 \quad \operatorname{codimBs}|H-\Pi|=3 .
$$

Therefore for a general divisor $P \in|H-\Pi|$ the effective cycle $Z_{P}=(Z \circ P)$ of codimension two is well defined and satisfies the inequality

$$
\operatorname{mult}_{o} Z_{P}=\operatorname{mult}_{o} Z+\operatorname{mult}_{\Pi} Z^{+}>8 n^{2} .
$$

Let $\Sigma_{P}=\left.\Sigma\right|_{P}$ be the restriction of the linear system $\Sigma$ onto $P$. Obviously, $\Sigma_{P} \subset\left|2 n H_{P}\right|$, where $H_{P}=\left.H\right|_{P}$ is the positive generator of the group Pic $P \cong \mathbb{Z}$, whereas the system $\Sigma_{P}$ is mobile (has no fixed components). The cycle $Z_{P}$ is the self-intersection of the system $\Sigma_{P}$ :

$$
Z_{P}=\left(D_{1} \circ D_{2}\right)
$$

where $D_{1}, D_{2} \in \Sigma_{P}$ are generic divisors. The variety $P$ is a hypersurface of degree $M$ in $\mathbb{P}^{M}$, which may have isolated singular points, but the point $o \in P$ itself is non-singular.

Proposition 4.5 The pair $\left(P, \frac{1}{n} \Sigma_{P}\right)$ is not log canonical at the point $o$, that is, it has a non log canonical singularity with the centre at that point. If the pair $\left(P, \frac{1}{n} \Sigma_{P}\right)$ has a non-canonical singularity with the centre $B \ni o, B \neq o$, then either $\operatorname{dim} B \leq 2$, or $B=\Delta=\mathrm{Bs}|H-\Pi|$ (and in the latter case the inequality mult ${ }_{\Delta} \Sigma>n$ ) holds.

Proof The first claim follows from the inversion of adjunction [14]. Let us consider the second one (it is not used in the subsequent proof). If $B \neq \Delta$, then $\operatorname{codim}(B \subset P) \geq 3$ (otherwise, by the genericity of $P$, the original system $\Sigma$ has a maximal subvariety of codimension 2 , which is not true by assumption). Therefore, the $4 n^{2}$-inequality holds:

$$
\operatorname{mult}_{B} Z_{P}>4 n^{2}
$$

Let $Q \in\left|H_{P}\right|$ be a general (in particular, everywhere non-singular) hyperplane section of $P$ and $Z_{Q}=\left(Z_{P} \circ Q\right)$. Then on $Q$ the cycle $Z_{Q} \sim 4 n^{2} H_{Q}$ of codimension two satisfies the inequality

$$
\text { mult }_{B \cap Q} Z_{Q}>4 n^{2}
$$

and $\operatorname{dim} B \cap Q \leq 1$ by Proposition 5 in [19]. Proof is complete.

Note that by the genericity of the hyperplane section $P$ the linear system $\Sigma_{P}$ satisfies the inequality

$$
v=\operatorname{mult}_{o} \Sigma_{P}\left(=\operatorname{mult}_{o} \Sigma\right) \leq 3 n .
$$


Now let $\Pi_{1} \subset \Pi_{2}$ be a generic pair of linear subspaces of dimensions 5 and 6 in $\mathbb{P}^{M}=\langle P\rangle$, containing the point $o$, and $X_{i}=P \cap \Pi_{i}$ the corresponding sections of the hypersurface $P$. By the inversion of adjunction the pair $\left(X_{i}, \frac{1}{n} \Sigma_{i}\right)$, where $\Sigma_{i}=\left.\Sigma_{P}\right|_{X_{i}}$, has the point $o$ as an isolated centre of a non $\log$ canonical singularity. Let $X_{i}^{+} \subset P^{+}$ be the strict transform of $X_{i}$, so that

$$
\varphi_{i}: X_{i}^{+} \rightarrow X_{i}
$$

is the blow up of the point $o \in X_{i}$ and $E^{(i)}=E \cap X_{i}^{+}$the exceptional divisor of the morphism $\varphi_{i}$. The pairs

$$
\square_{1}=\left(X_{1}^{+}, \frac{1}{n} \Sigma_{1}^{+}+\frac{v-3 n}{n} E^{(1)}\right)
$$

and

$$
\square_{2}=\left(X_{2}^{+}, \frac{1}{n} \Sigma_{2}^{+}+\frac{v-4 n}{n} E^{(2)}\right)
$$

are not $\log$ canonical (recall that $v \leq 3 n$ ) and satisfy the conditions of the connectedness principle with respect to the birational morphisms $\varphi_{1}$ and $\varphi_{2}$, respectively (see [14, Section 17.4]). The centre of any non $\log$ canonical singularity of the pair $\square_{i}$, intersecting $E^{(i)}$, is contained in $E^{(i)}$ (Proposition 4.5), so that we conclude that the union $L C S(\square)_{i}$ of centres of non log canonical singularities of the pair $\square_{i}$, intersecting $E^{(i)}$, is a connected closed subset in $E^{(i)}$. Recall that $E^{(1)} \cong \mathbb{P}^{3}$ and $E^{(2)} \cong \mathbb{P}^{4}$. For the pair $\square_{1}$ there are three options:

- $\operatorname{LCS}\left(\square_{1}\right)$ is a point $p \in E^{(1)}$,

- $\operatorname{LCS}\left(\square_{1}\right)$ is a connected curve,

- LCS $\left(\square_{1}\right)$ is a union of curves and surfaces, and in this union there is at least one surface.

For the pair $\square_{2}$ there are, respectively, four options, $\operatorname{dim} \operatorname{LCS}\left(\square_{2}\right) \in\{0,1,2,3\}$, and if $\operatorname{LCS}\left(\square_{2}\right)$ is zero-dimensional, then this set consists of one point.

Now looking at the pair

$$
\square_{12}=\left(X_{2}^{+}, \frac{1}{n} \Sigma_{2}^{+}+\frac{v-3 n}{n} E^{(2)}\right),
$$

we see that $\operatorname{LCS}\left(\square_{12}\right)$ is either a line in $E^{(2)} \cong \mathbb{P}^{4}$, or a connected union of surfaces (every hyperplane section of which is connected), or a union of surfaces and divisors in $E^{(2)}$. Since the pair $\square_{12}$ is obviously "more effective" than the pair $\square_{2}$, we have the inclusion

$$
\operatorname{LCS}\left(\square_{2}\right) \subset \operatorname{LCS}\left(\square_{12}\right),
$$

in particular, $\left(\operatorname{LCS}\left(\square_{2}\right) \cap X_{1}^{+}\right) \subset \operatorname{LCS}\left(\square_{1}\right)$. 
Now let us come back to the hypersurface $P$ and its blow up $\varphi_{P}: P^{+} \rightarrow P$ at the point $o$. From what was said, it follows that the pairs

$$
\square=\left(P^{+}, \frac{1}{n} \Sigma_{P}^{+}+\frac{v-3 n}{n} E_{P}\right)
$$

and

$$
\square^{*}=\left(P^{+}, \frac{1}{n} \Sigma_{P}^{+}+\frac{v-4 n}{n} E_{P}\right)
$$

are not log canonical, and moreover, one of the following six cases takes place.

Case 1.1. There are non $\log$ canonical singularities of the pairs $\square^{*}$ and $\square$, the centres of which on $P^{+}$are linear subspaces $\Theta \subset \Lambda \subset E_{P}$ of codimension 4 and 3 , respectively. Case 1.2. There exists a non $\log$ canonical singularity of the pair $\square^{*}$, the centre of which on $P^{+}$is a linear subspace $\Lambda \subset E_{P}$ of codimension 3 .

Case 2.1. There exist non log canonical singularities of the pairs $\square^{*}$ and $\square$, the centres of which on $P^{+}$are a linear subspace $\Theta \subset E_{P}$ of codimension 4 and an irreducible subvariety $B \subset E_{P}$ of codimension 2, respectively, where $\Theta \subset B$.

Case 2.2. There are non log canonical singularities of the pairs $\square^{*}$ and $\square$, the centres of which on $P^{+}$are irreducible subvarieties $B^{*} \subset B \subset E_{P}$ of codimension 3 and 2 , respectively.

Case 2.3. There is a non log canonical singularity of the pair $\square^{*}$, the centre of which on $P^{+}$is an irreducible subvariety $B \subset E_{P}$ of codimension 2.

Case 3. There is a non log canonical singularity of the pair $\square$, the centre of which on $P^{+}$is an irreducible subvariety $B \subset E_{P}$ of codimension 1 .

The six cases listed above correspond to three possible values of the integer $\operatorname{dim} \operatorname{LCS}\left(\square_{1}\right)$, taking into account the type of the set $\operatorname{LCS}\left(\square_{2}\right)$.

The last case is the simplest one.

Proposition 4.6 The case 3 does not realize: $\operatorname{codim}\left(B \subset E_{P}\right) \geq 2$.

Proof Assume the converse: $B \subset E_{P}$ is a prime divisor. We argue as in the proof of Proposition 4.1 in [23] or in [4]: for the self-intersection $Z_{P}$ of the system $\Sigma_{P}$, taking into account that the pair $\square$ is not $\log$ canonical at $B$, we obtain the estimate

$$
\operatorname{mult}_{o} Z_{P}>v^{2}+4\left(4-\frac{v}{n}\right) n^{2}=(v-2 n)^{2}+12 n^{2} \geq 12 n^{2} .
$$

Therefore, there is an irreducible subvariety $Y \subset P$ of codimension two, satisfying the inequality

$$
\frac{\text { mult }_{o}}{\operatorname{deg}} Y>\frac{3}{M} \text {. }
$$

However, this contradicts Proposition 4.3. Proposition 4.6 is shown. Q.E.D. 
Remark 4.2 Once again, we emphasize that Proposition 4.3 implies the inequality

$$
\operatorname{mult}_{o} Z_{P} \leq 12 n^{2}
$$

which we will use in the sequel without special references.

\subsection{The techniques of counting multiplicities: the aprioric estimates}

Following the standard procedure of the method of maximal singularities, let us obtain now bounds from below for the multiplicities of the cycle $Z_{P}$, improving the $8 n^{2}$ inequality. We call these estimates aprioric, because they do not make use the additional geometric information available in the cases 1.1-2.3. To exclude those cases, the aprioric estimates are not sufficient and we will need some additional work, which will be carried out in Sects. 4 and 5 .

Proposition 4.7 (i) If the case 1.1 takes place, then the following inequalities hold:

$$
\operatorname{mult}_{o} Z_{P}+\operatorname{mult}_{\Lambda} Z_{P}^{+}>12 n^{2}
$$

and mult $_{\Theta} Z_{P}>4 n^{2}$. If the case 1.2 takes place, then the following estimate holds:

$$
\operatorname{mult}_{\Lambda} Z_{P}^{+}>4 n^{2}
$$

(ii) If either of the cases 2.1 or 2.2 takes place, then the following inequality holds

$$
\operatorname{mult}_{o} Z_{P}+\operatorname{mult}_{B} Z_{P}^{+}>12 n^{2} \text {, }
$$

in addition in the case 2.1 the estimate mult $_{\Theta} Z_{P}^{+}>4 n^{2}$ and in the case 2.2 the estimate mult $_{B *} Z_{P}^{+}>4 n^{2}$ hold.

(iii) If the case 2.3 takes place, then the following inequality holds:

$$
\operatorname{mult}_{B} Z_{P}^{+}>4 n^{2}
$$

Proof All the inequalities, listed above, belong to one of the two types: the type (16) for a non log canonical singularity of the pair $\square$ and the type (17) for a singularity of the pair $\square^{*}$. The proofs for each of the two types are completely identical, and for this reason we will show only these two inequalities.

Let us prove the inequality (16). It is true under a weaker assumption that the pair $\square$ has a non canonical singularity, the centre of which is the subspace $\Lambda$. This is what we will assume. Let

$$
\begin{aligned}
& \sigma_{i, i-1}: P_{i} \rightarrow P_{i-1} \\
& \cup \quad \cup \\
& E_{i} \rightarrow B_{i-1}
\end{aligned}
$$


be the resolution of the non canonical singularity of the pair $\square$, where $P_{1}=P^{+}$, $\sigma_{1,0}=\varphi_{P}, E_{1}=E_{P}, \sigma_{2,1}$ is the blow up of the subvariety $\Lambda=B_{1}$, and in general, $B_{i-1}$ is the centre of the fixed non canonical singularity of the pair $\square$ on $P_{i-1}, E_{i}=$ $\sigma_{i, i-1}^{-1}\left(B_{i-1}\right)$ is the exceptional divisor, finally, $i=1, \ldots, K$ and $E_{K}$ realizes the fixed non canonical singularity. Let $\Gamma$ be the oriented graph of that resolution, that is, its set of vertices is the set of exceptional divisors

$$
E_{1}, \ldots, E_{K}
$$

and the vertices $E_{i}$ and $E_{j}$ are joined by an oriented edge (an arrow; notation: $i \rightarrow j$ ), if and only if $i>j$ and $B_{i-1}$ is contained in the strict transform $E_{j}^{i-1}$ of the exceptional divisor $E_{j}$ on $P_{i-1}$, see [17] or [21, Chapter 2], also [24, Chapter 2] for the details. By the symbol $p_{i j}$ we denote the number of paths from the vertex $E_{i}$ to the vertex $E_{j}$, if $i \neq j$; we set $p_{i i}=1$. The fact that $E_{K}$ realizes a non canonical singularity of the pair $\square$, means that the inequality of the Noether-Fano type holds:

$$
\sum_{i=1}^{K} p_{K i} v_{i}>n\left(3 p_{K 1}+\sum_{i=2}^{K} p_{K i} \delta_{i}\right),
$$

where $v_{i}=\operatorname{mult}_{B_{i-1}} \Sigma^{i-1}$, and $\delta_{i}=\operatorname{codim} B_{i-1}-1$ is the discrepancy of $E_{i}$ with respect to $P_{i-1}$. By linearity of the inequality (19) we may assume that $v_{K}>n$ (if $v_{K} \leq n$, then $E_{K-1}$ is a non canonical singularity of the pair $\square$ and $K$ can be replaced by $K-1)$. Set

$$
L=\max \left\{2 \leq i \leq K \mid \operatorname{codim} B_{i-1} \geq 3\right\} .
$$

The graph $\Gamma$ breaks into the lower part with the vertices $E_{1}, \ldots, E_{L}$ and the upper part with the vertices $E_{L+1}, \ldots, E_{K}$. Now let us the well known trick of removing arrows (see, for instance, [23, §4] or [24, Chapter 2] for the details): let us remove all arrows that go from the vertices of the upper part to the vertex $E_{1}$, if such arrows exist. This operation does not change the numbers $p_{K 2}, \ldots, p_{K}$, but, generally speaking, decreases the number of paths from $E_{K}$ to $E_{1}$. Set $p_{i}=p_{K i}$ for $i=2, \ldots, K$ and let $p_{1}$ be the number of paths from $E_{K}$ to $E_{1}$ in the modified graph. Since $v_{1} \leq 3 n$, the inequality (19) remains true:

$$
\sum_{i=1}^{K} p_{i} v_{i}>n\left(3 p_{1}+\sum_{i=2}^{K} p_{i} \delta_{i}\right)
$$

in addition, the modification of the graph $\Gamma$ yields the estimate

$$
p_{1} \leq \sum_{i=2}^{L} p_{i}
$$


Set $Z_{P}^{i}$ to be the strict transform of the cycle $Z_{P}$ on $P_{i}, i=1, \ldots, L$; in particular, $Z_{P}^{1}=Z_{P}^{+}$. Set also for $i=1, \ldots, L$

$$
m_{i}=\operatorname{mult}_{B_{i-1}} Z_{P}^{i-1}
$$

Applying the technique of counting multiplicities (see, for example [21, Proposition 2.11] or [24, Chapter 2]), we obtain the inequality

$$
\sum_{i=1}^{L} p_{i} m_{i} \geq \sum_{i=1}^{K} p_{i} v_{i}^{2}
$$

whence in the standard way (computing the minimum of the quadratic form $\sum p_{i} v_{i}^{2}$ on the hyperplane, which we obtain, replacing the inequality sign in (20) by the equality sign) we deduce the estimate

$$
\left(\sum_{i=1}^{K} p_{i}\right)\left(\sum_{i=1}^{L} p_{i} m_{i}\right)>\left(3 p_{1}+\sum_{i=2}^{K} p_{i} \delta_{i}\right)^{2} n^{2}
$$

Now set

$$
\Sigma_{0}=\sum_{\delta_{i}=3, i \geq 2} p_{i}, \quad \Sigma_{1}=\sum_{\delta_{i}=2} p_{i}, \quad \Sigma_{2}=\sum_{\delta_{i}=1} p_{i},
$$

so that, in particular, $p_{1} \leq \Sigma_{0}+\Sigma_{1}$. Taking into account that the multiplicities $m_{i}$ do not increase, we obtain the inequality

$$
\left(p_{1}+\Sigma_{0}+\Sigma_{1}+\Sigma_{2}\right)\left(p_{1} m_{1}+\left(\Sigma_{0}+\Sigma_{1}\right) m_{2}\right)>\left(3 p_{1}+3 \Sigma_{0}+2 \Sigma_{1}+\Sigma_{2}\right)^{2} n^{2} .
$$

Recall that $m_{1}=$ mult $_{o} Z_{P}$ and $m_{2}=$ mult $_{\Lambda} Z_{P}^{+}$are precisely the multiplicities, which we are interested in, and we prove the inequality $m_{1}+m_{2}>12 n^{2}$. By linearity in $m_{1}, m_{2}$ and the last inequality (that is, the inequality (16)) and the inequality (22), it is sufficient to check that the estimate (22) does not hold for $m_{1}=8 n^{2}, m_{2}=4 n^{2}$ and for $m_{1}=12 n^{2}, m_{2}=0$. Since $p_{1} \leq \Sigma_{0}+\Sigma_{1}$, it is sufficient to consider the first case. Setting in (22) $m_{1}=8 n^{2}$ and $m_{2}=4 n^{2}$, cancelling $n^{2}$ and moving everything to the right hand side, we obtain the inequality

$$
0>\Phi\left(p_{1}, \Sigma_{0}, \Sigma_{1}, \Sigma_{2}\right)
$$

where

$$
\Phi\left(s, t_{0}, t_{1}, t_{2}\right)=\left(s-t_{2}\right)^{2}+6 s t_{0}+5 t_{0}^{2}+4 t_{0} t_{1}+2 t_{0} t_{2}
$$

We obtained a contradiction, which proves the inequality (16). 
Now let us show the inequality (17). The arguments are completely similar to those above, with the only difference that the coefficient at $p_{1}$ in the Noether-Fano inequality is 4 , the elementary discrepancies can take four, not three values, that is, $\delta_{i} \in\{1,2,3,4\}$, so that there are, generally speaking, four groups of vertices of the graph $\Gamma$ and we must set

$$
\Sigma_{0}=\sum_{\delta_{i}=4, i \geq 2} p_{i}, \quad \Sigma_{1}=\sum_{\delta_{i}=3} p_{i}, \quad \Sigma_{2}=\sum_{\delta_{i}=2} p_{i}, \quad \Sigma_{3}=\sum_{\delta_{i}=1} p_{i},
$$

and the inequality $p_{1} \leq \Sigma_{0}+\Sigma_{1}+\Sigma_{2}$ holds. The technique of counting multiplicities gives the following estimate, which is similar to the inequality (22):

$$
\begin{aligned}
& \left(p_{1}+\Sigma_{0}+\Sigma_{1}+\Sigma_{2}+\Sigma_{3}\right)\left(p_{1} m_{1}+\left(\Sigma_{0}+\Sigma_{1}+\Sigma_{2}\right) m_{2}\right) \\
& >\left(4 p_{1}+4 \Sigma_{0}+3 \Sigma_{1}+2 \Sigma_{2}+\Sigma_{3}\right)^{2} n^{2} .
\end{aligned}
$$

Since $m_{1} \leq 12 n^{2}$, to prove the inequality (17) (which in the notations of the resolution of singularities takes the form of the inequality $m_{2}>4 n^{2}$ ), it is sufficient to check that the inequality (23) can not be true for $m_{1}=12 n^{2}$ and $m_{2}=4 n^{2}$. Substituting these values into (23), cancelling $n^{2}$ and moving everything to the right hand side, we get the inequality

$$
0>\Phi\left(p_{1}, \Sigma_{0}, \Sigma_{1}, \Sigma_{2}, \Sigma_{3}\right)
$$

where

$$
\Phi\left(s, t_{0}, t_{1}, t_{2}, t_{3}\right)=\left(2 s-t_{3}\right)^{2}+(\ldots)
$$

where in the brackets we have a quadratic form in $s, t_{0}, t_{1}, t_{2}, t_{3}$ with nonnegative coefficients. We obtained a contradiction, proving the inequality (17).

The remaining inequalities of Proposition 4.7 are shown word for word in the same way as the inequality (16) or (17), depending on the type of the inequality.

Proof of Proposition 4.7 is complete.

The further work, completing the proof of Theorem 1.5, is organized in the following way: we exclude the cases 1.1-2.3, inspecting all geometric possibilities.

\section{Infinitely near case: II-exclusion of the linear case}

In this section we prove that the cases 1.1 and 1.2 do not realize: it is sufficient to exclude the first one, which immediately implies that the second one is impossible.

\subsection{Decomposition of an effective cycle}

Let us forget for a moment about the proof of Theorem 1.5 and consider one very simple construction which will be used below many times. Let $X$ be an arbitrary algebraic 
variety, $Y \subset X$ an irreducible subvariety and $Z$ an effective cycle of codimension two on $X$. Assume first that $\operatorname{codim}(Y \subset X) \leq 2$, that is, $Y$ is a prime Weil divisor on $X$ or an irreducible subvariety of codimension two.

Definition 5.1 We say that the presentation

$$
Z=Z_{0}+Z_{1}
$$

is a $Y$-decomposition of the cycle $Z$, if both cycles $Z_{0}, Z_{1}$ are effective and an irreducible component of the cycle $Z$ is contained in $Z_{0}$ (respectively, in $Z_{1}$ ) if and only if it is contained in $Y$ (respectively, not contained in $Y$ ).

Assume now that $\operatorname{codim}(Y \subset X) \geq 3$.

Definition 5.2 We say that the presentation

$$
Z=Z_{0}+Z_{1}
$$

is a $Y$-decomposition of the cycle $Z$, if both cycles $Z_{0}, Z_{1}$ are effective and an irreducible component of the cycle $Z$ is contained in $Z_{0}$ (respectively, in $Z_{1}$ ) if and only if it does not contain $Y$ (respectively, does contain $Y$ ).

Note that the definitions are not symmetric.

Let us come back to the proof of Theorem 1.5.

\subsection{Restriction onto a hyperplane section}

The main result of this section is the following

Proposition 5.1 The case 1.1 does not take place.

Proof Assume the converse: the case 1.1 takes place. Our purpose is to get a contradiction. We will do it in several steps, since the case under consideration is the hardest of the six ones. We use both inequalities of Proposition 4.7 for the case 1.1 without special comments.

First of all, let us repeat the operation of restricting onto a hyperplane section that was used in Sect. 3.

Let $R \subset P$ be a general hyperplane section, such that:

- $o \in R$, the variety $R$ is non-singular at that point,

- the hyperplane $E_{R}=R^{+} \cap E_{P}$ in $E_{P}$ contains the subspace $\Lambda$.

Let us restrict the system $\Sigma_{P}$ onto $R$ and obtain a mobile linear system $\Sigma_{R}$ on the hypersurface $R \subset\langle R\rangle \cong \mathbb{P}^{M-1}$ with the self-intersection $Z_{R}=\left.Z_{P}\right|_{R}$, satisfying the estimates

$$
\operatorname{mult}_{o} Z_{R}+\operatorname{mult}_{\Lambda} Z_{R}^{+}>12 n^{2}
$$

and

$$
\operatorname{mult}_{\Theta} Z_{R}>4 n^{2}
$$


The advantage of this situation is that the subspaces $\Theta \subset \Lambda \subset E_{R}$ are of codimension 3 and 2, respectively. Let

$$
Z_{R}=Z_{0}+Z_{1}
$$

be the $T_{R}$-decomposition of the cycle $Z_{R}$, where $T_{R}=\left(T_{o} R\right) \cap R$ is the tangent hyperplane section at the point $o$. Set

$$
\begin{aligned}
& d_{0}=\frac{1}{M n^{2}} \operatorname{deg} Z_{0}, \quad d_{1}=\frac{1}{M n^{2}} \operatorname{deg} Z_{1}, \\
& \mu_{0}=\frac{1}{n^{2}} \text { mult }_{o} Z_{0}, \quad \mu_{1}=\frac{1}{n^{2}} \text { mult }_{o} Z_{1} .
\end{aligned}
$$

We obtain the equality

$$
d_{0}+d_{1}=4
$$

and the inequality

$$
\mu_{0}+\mu_{1}>8
$$

Furthermore, set $\lambda_{1}=\frac{1}{n^{2}}$ mult $_{\Lambda} Z_{1}^{+}$, where $Z_{1}^{+}$is the strict transform of the cycle $Z_{1}$ on $R^{+}$, so that the following inequality holds:

$$
\mu_{0}+\mu_{1}+\lambda_{1}>12
$$

Proposition 4.3 implies that the multiplicities $\mu_{i}$ can be estimated in terms of the degrees $d_{i}$ in the following way: for $M \geq 18$ the inequality

$$
\mu_{0} \leq 3 d_{0}
$$

holds, for $M \leq 17$ a weaker estimate is true:

$$
\mu_{0} \leq \frac{8 M}{3(M-2)} d_{0}
$$

Since none of the components of the cycle $Z_{1}$ is contained in the tangent section $T_{R}=\left\{\left.q_{1}\right|_{R}=0\right\}=R \cap T_{o} R$, by the part (ii) of Proposition 4.3 the inequality

$$
\mu_{1} \leq \frac{2 M}{M-2} d_{1}
$$

holds. Finally, it is obvious that the following estimate holds:

$$
\mu_{1} \geq \lambda_{1} .
$$


The system of six equations and inequalities (24-30) (it is six, because depending on whether $M \geq 18$ or $M \leq 17$, we choose the inequality (27) or (28)) forms the first system of relations for the five parameters introduced above.

Since $\left.q_{2}\right|_{\Theta} \not \equiv 0$, the components of the cycle $Z_{R}$, the strict transforms of which contain the linear subspace $\Theta$, can not be contained in $T_{R}$. For that reason, mult ${ }_{o} Z_{1} \geq$ mult $_{\Theta} Z_{1}^{+}>4 n^{2}$, so that the following inequality holds:

$$
\mu_{1}>4
$$

\subsection{Additional estimates for the cycle $Z_{1}$}

Now let us consider the cycle $Z_{1}$, the most important part of the self-intersection $Z_{R}$, since it contains the linear subspace $\Lambda$. First of all, none of the components of the cycle $Z_{1}$ is contained in the tangent section $T_{R}=R \cap T_{o} R$ and for that reason $\left(Z_{1} \circ T_{R}\right)$ is an effective cycle of codimension 2 on the hypersurface $T_{R}$. The latter has a quadratic singularity at the point $o$, so that

$$
\left\{\left.q_{2}\right|_{T_{o} R}=0\right\}
$$

is its tangent cone at that point. Its projectivization will be denoted by the symbol $Q_{R}$. Obviously,

$$
Q_{R}=T_{R}^{+} \cap E
$$

By the condition (R2) the intersection $\left[Q_{R} \cap \Lambda\right]$ is an irreducible quadric; it is subvariety of codimension two on $Q_{R}$. Now let us compute the multiplicity mult ${ }_{o}\left(Z_{1} \circ T_{R}\right)$. By the rules of the intersection theory (see [8] or [24, Chapter 2]), write

$$
\left(Z_{1}^{+} \circ T_{R}^{+}\right)=\left(Z_{1} \circ T_{R}\right)^{+}+Z_{Q},
$$

where $Z_{Q}$ is an effective divisor on the quadric $Q_{R}$ (outside $Q_{R}$ the effective cycles $\left(Z_{1}^{+} \circ T_{R}^{+}\right)$and $\left(Z_{1} \circ T_{R}\right)^{+}$obviously coincide). Now we have

$$
\operatorname{mult}_{o}\left(Z_{1} \circ T_{R}\right)=2 \mu_{1} n^{2}+\operatorname{deg} Z_{Q}
$$

Setting $\mu_{2}=\frac{1}{n^{2}} \operatorname{mult}_{o}\left(Z_{1} \circ T_{R}\right)$, and $\operatorname{deg} Z_{Q}=2 \delta n^{2}$, we obtain the equality

$$
\mu_{2}=2 \mu_{1}+2 \delta
$$

Obviously, $\operatorname{deg}\left(Z_{1} \circ T_{R}\right)=\operatorname{deg} Z_{1}$. Furthermore, set

$$
\lambda_{2}=\frac{1}{n^{2}} \operatorname{mult}_{\left[Q_{R} \cap \Lambda\right]}\left(Z_{1} \circ T_{R}\right)^{+} .
$$


Since the following inequality is obviously true:

$$
\operatorname{mult}_{\left[Q_{R} \cap \Lambda\right]}\left(Z_{1}^{+} \circ T_{R}^{+}\right) \geq \operatorname{mult}_{\Lambda} Z_{1}^{+}
$$

by Corollary 5.1, which is shown below, we get the estimate

$$
\lambda_{2} \geq \lambda_{1}-\delta
$$

Finally, since mult $_{o}\left(Z_{1} \circ T_{R}\right) \geq \operatorname{mult}_{\left[Q_{R} \cap \Lambda\right]}\left(Z_{1} \circ T_{R}\right)^{+}$, we obtain the estimate

$$
\mu_{2} \geq \lambda_{2}
$$

Now we have to take into account the input of the infinitely near subvariety $\left[Q_{R} \cap \Lambda\right]$.

Proposition 5.2 The following estimate holds:

$$
\mu_{2}+2 \lambda_{2} \leq 4 \frac{M}{M-3} d_{1}
$$

Proof Let $H_{R}$ be the class of a hyperplane section of the hypersurface $R \subset\langle R\rangle \cong$ $\mathbb{P}^{M-1}$. Consider the pencil $\left|H_{R}-\Lambda\right|$ of hyperplane sections of $R$, defined by the condition: for $S \in\left|H_{R}-\Lambda\right|$ we have $S^{+} \supset \Lambda$. The base set $\Delta_{R}$ of the pencil $\left|H_{R}-\Lambda\right|$ is an irreducible subvariety of codimension two in $T_{R}$, of codimension 3 in $R$; more precisely, $\Delta_{R} \subset\left\langle\Delta_{R}\right\rangle \cong \mathbb{P}^{M-3}$ is a hypersurface of degree $M$, where the linear span $\left\langle\Delta_{R}\right\rangle$ is determined by the condition

$$
\left\langle\Delta_{R}\right\rangle^{+} \cap E=\Lambda
$$

Now let $Y$ be an arbitrary subvariety of codimension 2 in $R$. For a general divisor $S \in\left|H_{R}-\Delta\right|$ we have $Y \not \subset S$, so that $(Y \circ S)$ is an effective cycle of codimension 3 on $R$. By construction,

$$
\operatorname{mult}_{o}(Y \circ S) \geq \operatorname{mult}_{o} Y+2 \operatorname{mult}_{\left[Q_{R} \cap \Lambda\right]} Y^{+}
$$

(since $\operatorname{deg}\left[Q_{R} \cap \Lambda\right]=2$ ). However, $S$ is a section of the singular hypersurface $V \cap T_{o} V$ by a linear subspace of codimension 3 , so that, applying the regularity condition (R1) and arguing in word for word the same way as in the proof of Proposition 4.3, by means of the technique of hypertangent divisors, applied to the cycle $(Y \circ S)$, we obtain the estimate

$$
\operatorname{mult}_{o} Y+2 \text { mult }_{\left[Q_{R} \cap \Lambda\right]} Y^{+} \leq \frac{4}{M-3} \operatorname{deg} Y
$$

(recall that on $S$ the cycle $(Y \circ S)$ has codimension 2, so that this cycle can be considered as an effective cycle of codimension 3 on a section of the hypersurface $V$ by a linear subspace of codimension 3 , which by the condition (R1) satisfies the regularity condition). 
The inequality (35) follows from (36) in a trivial way.

Proof of Proposition 5.2 is complete.

\subsection{On the multiplicities of subvarieties on a quadric}

Let us put off the proof of Theorem 1.5 and show the fact about multiplicities of subvarieties on a quadric hypersurface that was used in Sect. 4.3. Let $Q \subset \mathbb{P}^{N}$ be an irreducible quadric, $\operatorname{dim} \operatorname{Sing} Q=s_{Q}$, and $Y \subset X \subset Q$ irreducible subvarieties.

Proposition 5.3 Assume that the inequality

$$
\operatorname{dim} X+\operatorname{dim} Y>N+s_{Q}+1
$$

holds. Then the following estimate is true:

$$
\text { mult }_{Y} X \leq \frac{1}{2} \operatorname{deg} X
$$

Proof Assume the converse:

$$
\text { 2mult }_{Y} X>\operatorname{deg} X \text {. }
$$

By the assumption on the dimensions $Y \not \subset$ Sing $Q$. Take an arbitrary point $p \in$ $Y \backslash \operatorname{Sing} Q$.

Lemma 5.1 The variety $X$ is contained in the tangent hyperplane $T_{p} Q$.

Proof Assume the converse: $X \not \subset T_{p} Q$. Then the effective cycle $\left(X \circ\left(T_{p} Q \cap Q\right)\right)$ is well defined. Its degree is equal to $\operatorname{deg} X$ and its multiplicity at the point $p$ is at least 2 mult $_{p} X>\operatorname{deg} X$, which is impossible. Q.E.D. for the lemma.

Therefore, the following inclusion takes place

$$
X \subset \bigcap_{p \in Y \backslash \operatorname{Sing} Q} T_{p} Q .
$$

Proof of the proposition will be complete, if we show that the dimension of the right hand side of the inclusion is strictly smaller than $\operatorname{dim} X$. Note that $\operatorname{Sing} Q \subset \mathbb{P}^{N}$ is a linear subspace and for any non-singular point $p \in Q$ we have $\operatorname{Sing} Q \subset T_{p} Q$.

Consider the section $Q^{*}$ of the quadric $Q$ by a general linear subspace of codimension $s_{Q}+1$ (in particular, not meeting Sing $Q$ ). The quadric $Q^{*}$ is non-singular. Let $Y^{*} \subset X^{*}$ be the corresponding sections of the varieties $Y$ and $X$.

Obviously, $Y^{*}$ contains at least

$$
\operatorname{dim} Y^{*}=\operatorname{dim} Y-s_{Q}-1
$$


linearly independent points, so that the linear space

$$
\bigcap_{p \in Y^{*}} T_{p} Q^{*}
$$

has the dimension not higher than the number

$$
N-\left(s_{Q}+1\right)-\left(\operatorname{dim} Y-s_{Q}-1\right)=N-\operatorname{dim} Y .
$$

Therefore, $\operatorname{dim} X^{*}=\operatorname{dim} X-s_{Q}-1 \leq N-\operatorname{dim} Y$. However, by assumption the opposite inequality holds. This contradiction completes the proof of Proposition 5.3.

Corollary 5.1 Let $o \in V$ be an arbitrary point, $\Pi \subset T_{o} V$ a linear subspace of codimension two in the vector tangent space $T_{o} V \cong \mathbb{C}^{M}, \mathbb{P}(\Pi) \cong \mathbb{P}^{M-3}$ its projectivization. Let $X, Y$ be irreducible subvarieties of codimension 1 and 2 on the quadric hypersurface $Q=\left\{q_{2} \mid \mathbb{P}(\Pi)=0\right\} \subset \mathbb{P}(\Pi)$. Then the estimate (38) holds for $M \geq 16$.

Proof Obviously in the notations of Proposition 5.3 we have: $\operatorname{dim} X=M-5, \operatorname{dim} Y=$ $M-6, N=M-3$ and by the regularity condition (R2) the estimate

$$
s_{Q} \leq\left[\frac{1}{2}(\sqrt{8 M+1}-1)\right]+1
$$

holds. Therefore, the inequality (37) follows from the estimate $M-10>$ $\left[\frac{1}{2}(\sqrt{8 M+1}-1)\right]$ which holds for $M \geq 16$. Applying Proposition 5.3, we complete the proof.

\subsection{Exclusion of the linear case}

Let us complete the proof of Proposition 5.1. The six linear equations and inequalities (32-35) form the second system of relations for now $5+3=8$ parameters $d_{*}, \mu_{*}, \lambda_{*}$ and $\delta$. Joining the first and second systems of relations, adding to them the inequality (31), we obtain 11 linear equations and inequalities for 8 nonnegative real parameters. replacing the strict inequalities everywhere by the non-strict ones, we obtain $11+8=19$ linear equations and non-strict linear inequalities, defining some (obviously, compact) convex subset

$$
\Xi \subset \mathbb{R}^{8}
$$

Proposition 5.4 The set $\Xi$ is empty.

Proof It is sufficient to apply any computer program to solve a suitable problem of linear programming, for example

$$
\mu_{0} \rightarrow \max _{\Xi} .
$$

For MAPLE the corresponding command can be written in the following way: 


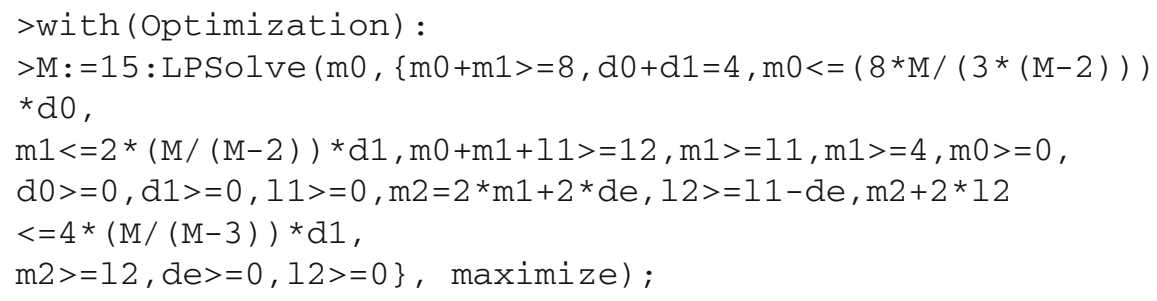

and its application gives the following result:

Error, (in Optimization:-LPSolve) no feasible solution found

Note that the inequalities $(28,29,35)$, that is, the only relations that depend on the parameter $M$ in the set of relations examined by the computer, get sharper as $M$ gets higher, so it is sufficient to consider the case $M=15$ : if $\Xi=\emptyset$ for $M=15$, then it is the more so empty for higher values of $M$. (This remark applies to other cases excluded with the help of the computer in the sequel.) This completes the proof of Proposition 5.4. (The convex set $\Xi$ is defined by 17 linear inequalities in the affine subspace of codimension two

$$
\left\{d_{0}+d_{1}=4, \mu_{2}=2 \mu_{1}+2 \delta\right\} \subset \mathbb{R}^{8},
$$

so that to solve the problem of linear programming, one needs to inspect a finite set of points of bounded cardinality. Each of these points is checked for being a point of the set $\Xi$. Therefore, the proof of Proposition 5.4 can be given over to the computer.)

Therefore, the first and second systems of relations, obtained above, define the empty set in $\mathbb{R}^{8}$. Therefore, the case 1.1 does not take place. Proposition 5.1 is shown. Q.E.D.

Corollary 5.2 The case 1.2 does not take place.

Proof Since a non log canonical singularity of the pair $\square^{*}$ is automatically a non log canonical singularity of the pair $\square$, the case 1.2 is a version of the case 1.1 (for $\Theta$ one can take any hyperplane in $\Lambda$ ). Q.E.D. for the corollary.

\section{Infinitely near case: III-exclusion of the non-linear case}

In this section we exclude the case 2 , which completes the exclusion of the infinitely near case (and so the proof of Theorem 1.5).

\subsection{The case 2.1, $B$ is not contained in a quadric}

Let us consider first the 2.1 and assume that the subvariety $B$ is not contained in any quadric hypersurface in $E_{P}$. (Note, that in the case 2.1 the subvariety $B$ is certainly not contained in the quadric $Q_{P}$, since $B \supset \Theta$ and $\Theta \not \subset Q_{P}$.) 
Proposition 6.1 The following inequality holds:

$$
\operatorname{5mult}_{B} \Sigma_{P}^{+} \leq 2 \operatorname{mult}_{o} \Sigma_{P}
$$

Proof Recall that the multiplicity mult $\Sigma_{P}$ is denoted by the letter $v$ and set $v_{B}=$ mult $_{B} \Sigma_{P}^{+}$. In terms of the resolution of the maximal singularity of the system $\Sigma_{P}$ we have $v=v_{1}$ and $v_{B}=v_{2}$. Assume that the opposite inequality holds: $5 v_{B}>2 v$. Let us show that this assumption leads to a contradiction with the mobility of the linear system $\Sigma_{P}^{+}$: the divisor $E_{P}$ can not be a fixed component of $\Sigma_{P}^{+}$, that is,

$$
E_{P} \not \subset \mathrm{Bs} \Sigma_{P}^{+}
$$

We will obtain a contradiction, thus proving the claim of our proposition.

Since the subvariety $B$ is not contained in any quadric hypersurface, its degree $\operatorname{deg} B$ (as a subvariety of the projective space $E_{P}$ ) is at least 5 .

Indeed, $d_{B}=\operatorname{deg} B \geq 3$. Furthermore, $B$ is not a cone over a curve: otherwise, $B$ contains a linear subspace of codimension 3 in $E_{P}$, which is excluded by the proof of Proposition 5.1. Now, projecting from a point of general position $p \in B$, we exclude the option $d_{B}=3$. If $d_{B}=4$ and $B$ has at least one singular point of multiplicity 2 or 3 , then $B$ is contained in a hyperplane or an irreducible quadric, contrary to the assumption. Since a non-singular projective subvariety of codimension 2 and degree 4 in $\mathbb{P}^{k}, k \geq 4$, is a complete intersection of two quadrics (this is a well known fact; see also [12]), then $B$ is a complete intersection of two quadrics, either, if $B$ is non-singular or is a cone over a subvariety of degree 4 and dimension $\geq 2$. We have inspected all options. Therefore, $d_{B} \geq 5$.

Let $\Pi \subset E_{P}$ be a 2-plane of general position, so that $B_{\Pi}=B \cap \Pi$ is a finite set, consisting of $d_{B} \geq 5$ distinct points. Let $R_{1}, \ldots, R_{m}$ be all irreducible hypersurfaces in $E_{P}$, containing $B$ and contained in $\operatorname{Bs} \Sigma_{P}^{+}$, if there are any. Then $\operatorname{deg} R_{i} \geq 3$ and the irreducible curves $R_{i} \cap \Pi$ are all irreducible curves in the plane $\Pi$, contained in $\operatorname{Bs} \Sigma_{P}^{+}$and containing at least one point of the finite set $B_{\Pi}$.

Lemma 6.1 Neither three points of the set $B_{\Pi}$ are collinear.

Proof Assume the converse: there are three distinct points $p_{1}, p_{2}, p_{3} \in B_{\Pi}$, lying on the line $L$. Since $v_{B}>n$ and $v \leq 3 n$, we obtain, that $L \subset \operatorname{Bs} \Sigma_{P}^{+}$. As we noted above, this is impossible. Q.E.D. for the lemma.

Now let us consider any 5 distinct points $p_{1}, \ldots, p_{5} \in B_{\Pi}$ and the unique conic $C \subset \Pi$, containing those points. As the plane $\Pi$ is generic, the conic $C$ is not contained in the base locus Bs $\Sigma_{P}^{+}$, therefore, the restriction $\Sigma_{C}=\left.\Sigma_{P}^{+}\right|_{C}$ is well defined. It is a linear series of degree $2 v$ with 5 base points of multiplicity $v_{B}$. Since $5 v_{B}>2 v$, we have $C \subset \operatorname{Bs} \Sigma_{P}^{+}$, which is impossible. Proof of Proposition 6.1 is complete.

Now we can apply the technique of counting multiplicities and estimate the multiplicity of the self-intersection $Z_{P}$ at the point $o$ and its strict transform $Z_{P}^{+}$along the subvariety $B$.

$$
\text { Set } \mu=\operatorname{mult}_{o} Z_{P}, \mu_{B}=\operatorname{mult}_{B} Z_{P}^{+} \text {. }
$$


Proposition 6.2 The following inequality holds:

$$
\mu+\mu_{B}>\frac{81}{5} n^{2}
$$

Proof As in the proof of Proposition 4.7, fix a maximal singularity, the centre of which on $P^{+}$is a subvariety $B$ and take its resolution. We use the standard notations, associated with the resolution. The graph $\Gamma$ is assumed to be modified, so that the inequality

$$
p_{1} \leq \Sigma_{0}=\sum_{i=2}^{L} p_{i}
$$

holds. We have the inequality of Noether-Fano type

$$
\sum_{i=1}^{K} p_{i} v_{i}>\left(3 p_{1}+2 \Sigma_{0}+\Sigma_{1}\right) n
$$

where $\Sigma_{1}=\sum_{i=L+1}^{K} p_{i}$, and, besides, we know that $v_{1} \leq 3 n$ and $5 v_{2} \leq 2 v_{1}$; the multiplicities $v_{i}$ do not increase,

$$
v_{2} \geq v_{3} \geq \cdots \geq v_{K}
$$

By the technique of counting multiplicities, taking into account the inequalities

$$
\operatorname{mult}_{B_{i}} Z_{P}^{i} \geq \operatorname{mult}_{B_{i+1}} Z_{P}^{i+1}
$$

we obtain the estimate

$$
p_{1} \mu+\Sigma_{0} \mu_{B} \geq \sum_{i=1}^{K} p_{i} v_{i}^{2}
$$

For $v_{1}=v$ fixed, the minimum of the right hand side of the latter inequality on the hyperplane

$$
\sum_{i=1}^{K} p_{i} v_{i}=\left(3 p_{1}+2 \Sigma_{0}+\Sigma_{1}\right) n
$$

is attained at $v_{2}=\cdots=v_{K}=\theta$, where the value $\theta$ is computed from the equation

$$
p_{1} v+\left(\Sigma_{0}+\Sigma_{1}\right) \theta=\left(3 p_{1}+2 \Sigma_{0}+\Sigma_{1}\right) n
$$


Therefore, the inequality

$$
p_{1} \mu+\Sigma_{0} \mu_{B}>p_{1} v^{2}+\left(\Sigma_{0}+\Sigma_{1}\right) \theta^{2}
$$

holds. On the other hand, the equality (41) can be re-written in the following way:

$$
\Sigma_{1}=\frac{3 n-v}{\theta-n} p_{1}+\frac{2 n-\theta}{\theta-n} \Sigma_{0} .
$$

Recall that $v$ and $\theta$ are connected by the inequality $5 \theta \leq 2 v$. As a result, we obtain that the sum $\mu+\mu_{B}$ is strictly higher than the minimum of the function $x+y$ on the interval, cut out by the inequalities

$$
x \geq v^{2}, \quad x \geq y, \quad y \geq 0
$$

on the line

$$
\left\{p_{1} x+\Sigma_{0} y=\Psi(\nu, \theta)\right\} \subset \mathbb{R}_{x, y}^{2},
$$

where

$$
\Psi(v, \theta)=p_{1}\left(v^{2}+\frac{3 n-v}{\theta-n} \theta^{2}+\frac{n \theta^{2}}{\theta-n} \Sigma_{0}\right)
$$

The more so, this minimum is strictly higher than the number

$$
v^{2}+\frac{n \theta^{2}}{\theta-n} \text {. }
$$

It is easy to check that the minimum of the function (43) on the triangle

$$
\{\theta>n, v \leq 3 n, 5 \theta \leq 2 \nu\} \subset \mathbb{R}_{\nu, \theta}^{2}
$$

is attained for $v=3 n, \theta=\frac{6}{5} n$ and is equal to $\frac{81}{5} n^{2}$. This completes the proof of Proposition 6.2.

The inequality (39) is so strong that it makes it possible to easily complete the exclusion of the case 2.1 (under the assumption that $B$ is not contained in any quadric hypersurface in $E_{P}$ ). Indeed, since $d_{B} \geq 5$, we have the inequality

$$
\mu \geq 5 \mu_{B}
$$

It is easy to check that it is incompatible with the inequalities (39) and $\mu \leq 12 n^{2}$. This excludes the case under consideration (that is, the case 2.1 under the assumption that $B$ is not contained in any quadric in $\left.E_{P}\right)$. 


\subsection{Case 2.1, $B$ is contained in a quadric, but not in a hyperplane}

Now let us consider the case 2.1 under the assumption that $B$ is contained in some quadric in $E_{P}$, but $\langle B\rangle=E_{P}$, that is, $B$ is not contained in any hyperplane in $E_{P}$.

Proposition 6.3 The following inequality holds:

$$
2 \text { mult }_{B} \Sigma_{P}^{+} \leq \operatorname{mult}_{o} \Sigma_{P}
$$

Proof Again we write $v_{B}=$ mult $_{B} \Sigma_{P}^{+}$and $v=$ mult $_{o} \Sigma_{P}$. Since $B$ is not contained in a hyperplane, $\operatorname{Sec}(B)=E_{P}$. Let $L$ be a general secant line of the variety $B$. Since the system $\Sigma_{P}^{+}$has no fixed components, for a general divisor $D \in \Sigma_{P}$ we have $L \not \subset D^{+}$. Therefore,

$$
2 v_{B} \leq \sum_{x \in L \cap B}\left(L \cdot D^{+}\right)_{x} \leq\left(L \cdot D^{+}\right)=v
$$

as we claimed. The proposition is shown.

Corollary 6.1 The following estimate is true: $v_{B} \leq \frac{3}{2} n$.

The following claim is an analog of Proposition 6.2 in the situation under consideration

Proposition 6.4 The following inequality holds:

$$
\mu+\mu_{B}>(10+2 \sqrt{2}) n^{2} .
$$

Proof is completely similar to the proof of Proposition 6.2 given above: we argue in word for word the same way and, recalling that $\mu>8 n^{2}$, we get that the value $\mu+\mu_{B}$ is strictly higher than the minimum of the function

$$
\max \left(v^{2}, 8 n^{2}\right)+\frac{n \theta^{2}}{\theta-n}
$$

on the triangle

$$
\{\theta>n, v \leq 3 n, 2 \theta \leq v\} \subset \mathbb{R}_{v, \theta}^{2} .
$$

This minimum is attained for $v=2 \sqrt{2} n, \theta=\sqrt{2} n$ and is equal to $(10+2 \sqrt{2}) n^{2}$, which is what we need. Q.E.D.

Remark 6.1 Since $10+2 \sqrt{2} \approx 12.8$, the inequality (44) is considerably sharper than the aprioric inequality (18).

The estimate (44) is essentially weaker than (39), however, this is compensated by the additional geometric information about the subvariety $B$ : we know that $B \subset Q^{*}$, where $Q^{*} \neq Q_{P}$ is some irreducible quadric, and moreover by assumption $B$ is not a hyperplane section of the quadric $Q^{*}$. 
Lemma 6.2 The degree of the subvariety $B$ is at least 4 .

Proof We must exclude the option $d_{B}=\operatorname{deg} B=3$. Assume that this is the case. Then the rank of the quadratic form, defining $Q^{*}$, is equal to 3 or 4 , so that $B$ is swept out by a one-dimensional family of linear subspaces of codimension 3 in $E_{P}$. Proposition 5.1 excludes this situation. Q.E.D. for the lemma.

Corollary 6.2 The following inequality holds:

$$
\mu \geq 4 \mu_{B}
$$

Now we exclude the case under consideration in the same way as we used to exclude the case 1.1, with some simplifications. Let $Z_{P}=Z_{0}+Z_{1}$ be the $T_{P}$-decomposition of the cycle $Z_{P}$. Since $B \not \subset Q_{P}=T_{P}^{+} \cap E_{P}$, we have mult $Z_{1}^{+}=\mu_{B}$. Now, introducing the normalized parameters $d_{i}, \mu_{i}, i=0,1$, and $\lambda_{1}$, we obtain for them the system of the following inequalities: (24), (25), instead of (27) and (28) we have the estimate

$$
\mu_{0} \leq \max \left(3, \frac{8 M}{3(M-1)}\right) d_{0},
$$

instead of (29) we have the estimate

$$
\mu_{1} \leq \frac{2 M}{M-1} d_{1}
$$

finally, instead of (26) we have the estimate

$$
\mu_{0}+\mu_{1}+\lambda_{1}>10+2 \sqrt{2}
$$

and instead of (30) the stronger estimate

$$
\mu_{1} \geq 4 \lambda_{1}
$$

Using MAPLE, it is easy to check that this system of linear equations and inequalities has no solutions already for $M \geq 5$. This completes the exclusion of the case 2.1 under the assumption that $\langle B\rangle=E_{P}$.

\subsection{The case 2.1, $B$ is contained in a hyperplane}

Assume that $B$ is contained in some hyperplane $\Pi \subset E_{P}$. By Proposition 5.1, $B$ is a hypersurface of degree $d_{B} \geq 2$ in $\Pi$. Consider the linear system $\left|H_{P}-\Pi\right|$, that is, the pencil of hyperplane sections, the base set of which is the intersection $\Delta$ of the tangent section $T_{P}$ with the hyperplane in $\langle P\rangle$ that has $\Pi$ as the tangent cone. Let $Z_{P}=Z_{0}+Z_{1}$ be such a decomposition of the cycle $Z_{P}$, that $Z_{P}^{+}=Z_{0}^{+}+Z_{1}^{+}$is the $B$-decomposition of the effective cycle $Z_{P}^{+}$. 
Let $R \in\left|H_{P}-\Pi\right|$ be a general divisor. For the effective cycle $\left(Z_{1} \circ R\right)$ we have:

$$
\begin{aligned}
& \operatorname{deg}\left(Z_{1} \circ R\right)=\operatorname{deg} Z_{1}, \\
& \operatorname{mult}_{o}\left(Z_{1} \circ R\right) \geq \operatorname{mult}_{o} Z_{1}+2 \operatorname{mult}_{B} Z_{1}^{+},
\end{aligned}
$$

since $d_{B} \geq 2$. However, in the case under consideration $\left(Z_{1} \circ R\right)$ is an effective cycle of codimension two on the hyperplane section $R$, which itself satisfies the regularity conditions, and for that reason the inequality

$$
\frac{\text { mult }_{o}}{\operatorname{deg}}\left(Z_{1} \circ R\right) \leq \max \left(\frac{3}{M}, \frac{8}{3(M-2)}\right)
$$

holds; the right hand side for $M \geq 18$ does not exceed $3 / M$. Taking into account that mult $_{o} Z_{0} \leq \frac{3}{M} \operatorname{deg} Z_{0}$, we obtain a contradiction with the aprioric inequality (18). This excludes the case under consideration for $M \geq 18$.

Remark 6.2 In the argument given above we used the fact that $B \not \subset Q_{P}$ : it is for that reason that the scheme-theoretic intersection $\left(Z_{1} \circ R\right)$ is well defined. However, if $B \subset Q_{P}$, then $B=\Pi \cap Q_{P}$. Set $\Delta=\mathrm{Bs}\left|H_{P}-\Pi\right|$ (see above). Obviously,

$$
\operatorname{deg} \Delta=M, \quad \operatorname{mult}_{o} \Delta=2
$$

(because $\Delta^{+} \cap E_{P}=B$ ), so that writing

$$
Z_{1}=a \Delta+Z_{*}
$$

where $a \in \mathbb{Z}_{+}$and $Z_{*}$ does not contain $\Delta$ as a component, we repeat the previous argument and come to a contradiction for $M \geq 18$.

If we use all the information available, we can exclude the case under consideration for smaller values of $M$ as well. Namely, write $Z_{P}=Z_{0}+Z_{1}$, where $Z_{P}^{+}=Z_{0}^{+}+Z_{1}^{+}$ is the $\Theta$-decomposition of the effective cycle $Z_{P}^{+}$. The cycle $\left(Z_{1} \circ R\right)$ is well defined for a general divisor $R \in\left|H_{P}-\Pi\right|$. Furthermore, write

$$
\left(Z_{1} \circ R\right)=Z_{10}+Z_{11}
$$

where the strict transform of this equality on $P^{+}$is the $\Theta$-decomposition of the cycle $\left(Z_{1} \circ R\right)^{+}$. The cycle $Z_{10}$ satisfies the estimate

$$
\frac{\text { mult }_{o}}{\operatorname{deg}} Z_{10} \leq \frac{8}{3(M-2)} d_{10},
$$

but for $Z_{11}$ a much stronger inequality holds:

$$
\frac{\operatorname{mult}_{o}}{\operatorname{deg}} Z_{11} \leq \frac{2}{M-2} d_{11},
$$


since none of the components of the cycle $Z_{11}$ is contained in the tangent section $T_{R}=R \cap T_{o} R$, so that we can form the effective cycle $\left(Z_{11} \circ T_{R}\right)$ and then apply to this cycle of codimension two on $T_{R}$ the technique of hypertangent divisors. Finally, setting

$$
\xi_{1}=\frac{1}{n^{2}} \operatorname{mult}_{\Theta} Z_{P}^{+}, \quad \xi_{2}=\frac{1}{n^{2}} \operatorname{mult}_{\Theta} Z_{11}^{+},
$$

we get the following system of linear equations and inequalities: (24)-(27), and also

$$
\begin{aligned}
& \mu_{10}+\mu_{11}=\mu_{1}+2 \lambda_{1}+\delta_{1}, \quad \xi_{1}>4 \\
& d_{10}+d_{11}=d_{1}, \quad \xi_{2} \geq \xi_{1}-\delta_{1}, \quad \mu_{11} \geq \xi_{2} \\
& \mu_{10} \leq \frac{8 M}{3(M-2)} d_{10}, \quad \mu_{11} \leq \frac{2 M}{(M-2)} d_{11}
\end{aligned}
$$

Applying MAPLE we see that this system is incompatible (even when we replace all strict inequalities by the non-strict ones) already for $M \geq 11$. This completes the exclusion of the case 2.1 .

\subsection{The case 2.2, $B$ is not contained in $Q_{P}$}

Now assume that the case 2.2 takes place, where $B \not \subset Q_{P}$. Now, if $B$ is not contained in a quadric, we obtain a contradiction, arguing as in Sect. 5.1. If $B$ is contained in a quadric, but not contained in a hyperplane, then we obtain a contradiction, arguing as in Sect. 5.2. Therefore we assume that $B \subset \Pi$, where $\Pi \subset E_{P}$ is some hyperplane. Now, if $M \geq 18$ or if $B^{*} \not \subset Q_{P}$, then we obtain a contradiction in word for word the same way as in Sect. 5.3. Therefore we assume that $M \leq 17$ and $B^{*} \subset Q_{P}$ is a subvariety of codimension 2 .

Let us consider the pencil of hyperplane sections $\left|H_{P}-\Pi\right|$. Its base set $\Delta=$ $\mathrm{Bs}\left|H_{P}-\Pi\right|$ is a hyperplane section of the tangent section $T_{P}$. Write

$$
Z_{P}=a \Delta+Z_{*}
$$

where $a \in \mathbb{Z}_{+}$and $Z_{*}$ does not contain $\Delta$ as a component. For the subvariety $\Delta$ we have:

$$
\operatorname{deg} \Delta=M, \quad \operatorname{mult}_{o} \Delta=2, \operatorname{mult}_{B} \Delta^{+}=0
$$

and mult $B^{*} \Delta^{+}=1$. Therefore for the cycle $Z_{*}$ we have: $\operatorname{deg} Z_{*}=\left(4 n^{2}-a\right) M$,

$$
\operatorname{mult}_{o} Z_{*}=\operatorname{mult}_{o} Z_{P}-2 a>8 n^{2}-2 a, \quad \operatorname{mult}_{B} Z_{*}^{+}=\operatorname{mult}_{B} Z_{P}^{+}
$$

and mult $_{B^{*}} Z_{*}^{+}=$mult $_{B^{*}} Z_{P}^{+}-a>4 n^{2}-a$. Now let $R \in\left|H_{P}-\Pi\right|$ be a general divisor. By construction, $R$ does not contain irreducible components of the cycle $Z_{*}$ and for that reason the effective cycle $\left(Z_{*} \circ R\right)$ of codimension 2 on $R$ is well defined. Let $Z_{R}=\left(Z_{*} \circ R\right)=Z_{0}+Z_{1}$ be the $T_{R}$-decomposition of the cycle $Z_{R}$. 
Let $Q_{R}=Q_{P} \cap \Pi=T_{R}^{+} \cap E_{P}$ be the (projectivized) tangent cone to $T_{R}$, a quadric in $E_{R}=R^{+} \cap E_{P}=\Pi$. The subvariety $B^{*}$ is a prime divisor on $Q_{R}$ and for that reason is cut out on $Q_{R}$ by a hypersurface in $E_{R}$ of degree $\delta^{*} \geq 1$, so that $2 \delta^{*}=d^{*}=\operatorname{deg} B^{*}$.

Proposition 6.5 The equality $\delta^{*}=1$ holds, that is, $B^{*}$ is a hyperplane section of $Q_{R}$.

Proof Assume the converse: $\delta^{*} \geq 2$. In that case $d^{*} \geq 4$. Therefore, the inequality

$$
\text { mult }_{o} Z_{R} \geq 4 \text { mult }_{B^{*}} Z_{R}^{+}
$$

holds. To compute the left hand part, write

$$
\left(Z_{*}^{+} \circ R^{+}\right)=Z_{R}^{+}+\beta B+N,
$$

where $N$ is an effective divisor on $\Pi$, not containing $B$ as a component and $\beta \geq \mu_{B}$. By the intersection theory,

$$
\operatorname{mult}_{o} Z_{R}=\operatorname{mult}_{o} Z_{*}+\beta d_{B}+d_{N},
$$

where $d_{N}=\operatorname{deg} N$. On the other hand, by assumption $B^{*}$ is not contained in a hyperplane $\Pi$, that is, $\left\langle B^{*}\right\rangle=\Pi$ and for that reason the inequalities

$$
2 \text { mult }_{B^{*}} N \leq d_{N} \quad 2 \text { mult }_{B^{*}} B \leq d_{B}
$$

hold. Therefore, we have the estimate

$$
\operatorname{mult}_{B^{*}} Z_{R}^{+} \geq \operatorname{mult}_{B^{*}} Z_{*}^{+}-\frac{1}{2} \beta d_{B}-\frac{1}{2} d_{N} .
$$

Besides, we remember that the inequalities

$$
\frac{\text { mult }_{o}}{\operatorname{deg}} Z_{0} \leq \frac{8}{3(M-2)} \text { and } \frac{\text { mult }_{o}}{\operatorname{deg}} Z_{1} \leq \frac{2}{M-2}
$$

hold, and also the inequalities $\mu>8 n^{2}$ and $\mu+\mu_{B}>12 n^{2}$. Using MAPLE, it is easy to check (replacing, as usual, strict inequalities by non-strict ones), that the system of linear equations and inequalities, obtained above, has no solutions for $M \geq 13$. Proof of Proposition 6.5 is complete.

Therefore, $B^{*}=\Theta \cap Q_{R}$, where $\Theta \subset \Pi=E_{R}$ is a hyperplane. Instead of the inequality (45) we have a weaker estimate

$$
\operatorname{mult}_{o} Z_{R} \geq 2 \text { mult }_{B^{*}} Z_{R}^{+}
$$

and it is no longer sufficient to obtain a contradiction. Let us consider the linear system $\left|H_{R}-\Theta\right|$ on $R$ and set $\Delta^{*}=\mathrm{Bs}\left|H_{R}-\Theta\right|$ to be its base set (a divisor on the 
tangent section). By the regularity conditions we have mult $B^{*}\left(\Delta^{*}\right)^{+}=1$. Write down $Z_{0}=c \Delta^{*}+Z_{\sharp}$, where $c \in \mathbb{Z}_{+}$and $Z_{\sharp}$ does not contain $\Delta^{*}$ as a component. For a general divisor $D \in\left|H_{R}-\Theta\right|$ the effective cycle $\left(D \circ Z_{\sharp}\right)$ of codimension two on $T_{R}$ is well defined and satisfies the inequalities

$$
\operatorname{mult}_{o}\left(D \circ Z_{\sharp}\right) \geq \operatorname{mult}_{o} Z_{\sharp}+2 \text { mult }_{B^{*}} Z_{\sharp}^{+}
$$

and

$$
\frac{\text { mult }_{o}}{\operatorname{deg}}\left(D \circ Z_{\sharp}\right) \leq \frac{4}{M-2} .
$$

Adding the corresponding normalized inequalities to the previous ones and using MAPLE, we see that for $M \geq 13$ the case under consideration is impossible.

This completes the exclusion of the case 2.2 under the assumption that $B \not \subset Q_{P}$.

\subsection{The case 2.2, $B$ is contained in $Q_{P}$}

Assume that $B \subset Q_{P}$. Note, first of all, that $B$ is not contained in a hyperplane (that is, it is not a hyperplane section of $Q_{P}$ ): such an option is excluded by word for word the same arguments as those that were used in the case $B \not \subset Q_{P}, B \subset \Pi$, where $\Pi \subset E_{P}$ is a hyperplane. In particular, $d_{B} \geq 4$ and the estimate (44) holds.

Proposition 6.6 The subvariety $B^{*} \subset Q_{P}$ of codimension two is contained in a hyperplane $\Pi \subset E_{P}$.

Proof Assume the converse. Let $\Lambda \subset Q_{P}$ be a general linear subspace of maximal dimension, $B_{\Lambda}^{*}=B^{*} \cap \Lambda \subset \Lambda$ an irreducible subvariety of codimension two. For the linear $\operatorname{span}\left\langle B_{\Lambda}^{*}\right\rangle$ there are three options:

1. $\left\langle B_{\Lambda}^{*}\right\rangle=\Lambda$,

2. $\left\langle B_{\Lambda}^{*}\right\rangle$ is a hyperplane in $\Lambda$,

3. $\left\langle B_{\Lambda}^{*}\right\rangle=B_{\Lambda}^{*}$ is a subspace of codimension two in $\Lambda$.

Note at once, that the third option does not realize: (3) implies that $\operatorname{deg} B^{*}=2$ and then $B^{*}$ is contained in a hyperplane, contrary to our assumption.

Furthermore, $\operatorname{Sec}\left(B_{\Lambda}^{*}\right)=\left\langle B_{\Lambda}^{*}\right\rangle$. Set

$$
W=\overline{\bigcup_{\Lambda \subset Q_{P}}\left\langle B_{\Lambda}^{*}\right\rangle} .
$$

It follows from what was said that either $W$ is an irreducible divisor on $Q_{P}$, or $W=$ $Q_{P}$. However, in the first case $W \cap \Lambda$ is an irreducible hypersurface in $\Lambda$ (for a general $\Lambda$ ) and for that reason $W \cap \Lambda=\left\langle B_{\Lambda}^{*}\right\rangle$ is a hyperplane in $\Lambda$, and then $W$ is a hyperplane section of the quadric $Q_{P}$, where $B^{*} \subset W$, contrary to our assumption. Therefore, $W=Q_{P}$. From here we get the following fact. 
Lemma 6.3 For any effective divisor $Y$ on the quadric $Q_{P}$ the inequality

$$
\operatorname{deg} Y \geq 4 \text { mult }_{B^{*}} Y
$$

holds (the degree $\operatorname{deg} Y$ is understood as the degree of an effective cycle of codimension 2 on $\left.E_{P}\right)$.

Proof Denote by the symbol $H_{Q}$ the class of a hyperplane section of the quadric $Q_{P}$, so that $Y \sim \gamma H_{Q}$ for some $\gamma \geq 1$, where $\operatorname{deg} Y=2 \gamma$. Let $\Lambda$ be a general linear subspace of maximal dimension on $Q_{P}$ and $L \subset \Lambda$ a general secant line of the variety $B_{\Lambda}^{*}$. Since the lines $L$ sweep out $Q_{P}$, we may assume that $L \not \subset|Y|$. Let $x, y \in B_{\Lambda}^{*}$ be general points, where $L=[x, y]$. We have

$$
(L \cdot Y)_{Q_{P}}=\gamma \geq(L \cdot Y)_{x}+(L \cdot Y)_{y} \geq 2 \operatorname{mult}_{B^{*}} Y
$$

when the claim of the lemma follows. Q.E.D.

Now let $Z_{P}=Z_{0}+Z_{1}$ be, as usual, the $T_{P}$-decomposition of the cycle $Z_{P}$. Setting $\lambda_{i}=\frac{1}{n^{2}}$ mult $_{B} Z_{i}, i=0,1$, we obtain the following system of linear equations and inequalities: $(24,25,27)$, and also the estimate

$$
\mu_{0}+\mu_{1}+\lambda_{0}+\lambda_{1}>10+2 \sqrt{2}
$$

instead of (26), and also the estimates

$$
\begin{aligned}
& 2 \mu_{1}+d_{B} \lambda_{1} \leq \frac{4 M}{M-1} d_{1}, \\
& \mu_{0} \geq d_{B} \lambda_{0}, \quad \mu_{1} \geq d_{B} \lambda_{1} .
\end{aligned}
$$

Now set $\xi_{i}=\frac{1}{n^{2}}$ mult $_{B^{*}} Z_{i}^{+}, i=0,1$. By the lemma shown above, the estimate

$$
\mu_{0} \geq 4 \xi_{0}
$$

holds, besides, $\mu_{1} \geq \xi_{1}$ and, as we know, $\xi_{0}+\xi_{1}>4$. The inequality (47) can be sharpened. Write down

$$
\left(Z_{1}^{+} \circ T_{P}\right)=\left(Z_{1} \circ T_{P}\right)^{+}+N
$$

where $N$ is an effective divisor on the quadric $Q_{P}$. Set $d_{N}=\frac{1}{n^{2}} \operatorname{deg} N$, then we get

$$
d_{N} \geq d_{B} \lambda_{1}
$$

and the estimate

$$
2 \mu_{1}+d_{N} \leq \frac{4 M}{M-1} d_{1}
$$


holds. Setting $\xi_{N}=\frac{1}{n^{2}}$ mult $_{B^{*}} N$ and applying Lemma 6.3, we obtain the inequality

$$
d_{N} \geq 4 \xi_{N}
$$

Obviously,

$$
\frac{1}{n^{2}} \text { mult }_{B^{*}}\left(Z_{1} \circ T_{P}\right)^{+} \geq \xi_{1}-\xi_{N},
$$

so that, applying Lemma 6.3 once again, we get the inequality

$$
2 \mu_{1}+d_{N} \geq 4\left(\xi_{1}-\xi_{N}\right)
$$

Using MAPLE, we check that the system of linear equations and inequalities, obtained above, is incompatible. Q.E.D. for Proposition 6.6.

\subsection{Exclusion of the case 2.2}

Now let us assume that the hyperplane $\Pi \supset B^{*}$ is the only hyperplane in $E_{P}$ with that property, that is, $B^{*}$ is not the intersection of $Q_{P}$ with a linear subspace $\Theta \subset E_{P}$ of codimension two. In particular, $d^{*}=\operatorname{deg} B^{*} \geq 4$. Let $R \in\left|H_{P}-\Pi\right|$ be a general divisor of the pencil. Write down $Z_{P}=a \Delta+Z_{*}$, where $\Delta=\mathrm{Bs}\left|H_{P}-\Pi\right|, a \in \mathbb{Z}_{+}$ and $Z_{*}$ does not contain $\Delta$ as a component. To simplify the formulas, we will assume that $a=0$ and $Z_{*}=Z_{P}$ : if $a \geq 1$, then the system of linear equations and inequalities, obtained below, remains incompatible, which is easy to check.

So $Z_{P}=Z_{0}+Z_{1}$ is the $T_{P}$-decomposition of the cycle $Z_{P}$ and $\Delta$ is not an irreducible component of the cycle $Z_{0}$. Setting, as usual,

$$
\mu_{i}=\frac{1}{n^{2}} \operatorname{mult}_{o} Z_{i}, \quad \lambda_{i}=\frac{1}{n^{2}} \operatorname{mult}_{B} Z_{i}^{+},
$$

and $d_{i}=\frac{1}{M n^{2}} \operatorname{deg} Z_{i}, i=0,1$, we obtain the standard set of linear equations and inequalities: $(24,25,46)$, and also the inequalities (27), (48) with $d_{B}=4$ and the estimate

$$
\mu_{1} \leq \frac{2 M}{M-1} d_{1}
$$

Set $\xi_{i}=\frac{1}{n^{2}}$ mult $_{B^{*}} Z_{i}^{+}, i=0,1$. In our case $\xi_{0}+\xi_{1}>4$.

Lemma 6.4 The following inequality holds:

$$
\mu_{0} \geq 2\left(\lambda_{0}+\xi_{0}\right)
$$

Proof $Z_{0}^{+}$is an effective divisor on $T_{P}^{+}$, and its projectivized tangent cone $Z_{0}^{+} \cap E_{P}$ is an effective divisor on the quadric $Q_{P}$. Let $\Lambda \subset Q_{P}$ be a general linear subspace. 
Let $p \in B^{*} \cap \Lambda$ and $q \in B \cap \Lambda$ be points of general position. The lines $L=[p q]$ sweep out $\Lambda$ and for that reason we may assume that $L \not \subset Z_{0}^{+}$. Therefore, for the intersection numbers on $Q_{P}$ we have:

$\frac{1}{2} \mu_{0} n^{2}=\left(L \cdot\left(Z_{0}^{+} \cap E_{P}\right)\right)_{Q_{P}} \geq\left(L \cdot\left(Z_{0}^{+} \cap E_{P}\right)\right)_{p}+\left(L \cdot\left(Z_{0}^{+} \cap E_{P}\right)\right)_{q} \geq\left(\xi_{0}+\lambda_{0}\right) n^{2}$,

which is what we claimed. Q.E.D. for the lemma.

Now write down

$$
\left(Z_{i}^{+} \circ R^{+}\right)=\left(Z_{i} \circ R\right)^{+}+N_{i}
$$

where $N_{1}$ is an effective divisor on $\Pi$, and $N_{0}=c\left(\Pi \cap Q_{P}\right), c \in \mathbb{Z}_{+}$. Since $\left\langle B^{*}\right\rangle=\Pi$, the inequality

$$
\operatorname{deg} N_{i} \geq 2 \text { mult }_{B^{*}} N_{i}
$$

holds (for $i=0$ it is the equality, since obviously mult $B^{*} N_{0}=1$ ). Setting

$$
\zeta_{i}=\frac{1}{n^{2}} \operatorname{mult}_{B^{*}}\left(Z_{i} \circ R\right)^{+},
$$

we obtain inequalities

$$
\zeta_{i} \geq \xi_{i}-\frac{1}{2} n_{i}
$$

where $n_{i}=\frac{1}{n^{2}} \operatorname{deg} N_{i}$. Setting $\alpha_{i}=\frac{1}{n^{2}}$ mult $_{o}\left(Z_{i} \circ R\right), i=0,1$, we obtain the set of standard estimates

$$
\alpha_{i} \geq \mu_{i}+n_{i}, \quad \alpha_{0} \leq \max \left(3, \frac{8 M}{3(M-2)}\right) \cdot d_{0}, \quad \alpha_{i} \geq 4 \zeta_{i}, i=0,1
$$

(the last is true by the inequality $\operatorname{deg} B^{*} \geq 4$, as $\left\langle B^{*}\right\rangle=\Pi$ ). Besides, one more important inequality holds.

Lemma 6.5 The following estimate holds:

$$
4 n_{0} \geq 4 \xi_{0}-\mu_{0}
$$

Proof Once again, let $\Lambda \subset Q_{P}$ be a general linear subspace of the maximal dimension and $L$ a general secant line of the variety $B^{*} \cap \Lambda$. The lines $L$ sweep out the hyperplane section $\Pi \cap Q_{P}$ and for that reason it is sufficient to show the inequality

$$
\beta=\frac{1}{n^{2}} \text { mult }_{L} Z_{0}^{+} \geq \frac{1}{4}\left(2 \xi_{0}-\frac{1}{2} \mu_{0}\right)
$$

Since $n_{0} \geq 2 \beta$, the inequality (50) implies the claim of our lemma. 
Consider a general line $L^{*} \subset \Lambda$, intersecting $L$, and let $S \ni o$ be a generic twodimensional germ of an isolated quadratic singularity at the point $o, S \subset T_{P}$, such that $S^{+} \cap E_{P}=L+L^{*}$, and $S^{+}$is a non-singular surface. Obviously,

$$
\left.Z_{0}^{+}\right|_{S^{+}} \sim-\left.\left(\frac{1}{2} \operatorname{mult}_{o} Z_{0}\right) E_{P}\right|_{S^{+}},
$$

whereas the effective 1-cycle $\left.Z_{0}^{+}\right|_{S^{+}}$has the line $L$ as a component of the multiplicity $\beta n^{2}$. Taking this component out, we obtain that the effective 1-cycle

$$
C=\left(\left.Z_{0}^{+}\right|_{S^{+}}-\beta n^{2} L\right)
$$

does not have $L$ as a component, and its multiplicity at two distinct points $p, q \in L$ is at least

$$
\left(\xi_{0}-\beta\right) n^{2}
$$

Computing the intersection $(C \cdot L)$, we obtain the inequality

$$
\frac{1}{2} \mu_{0}+2 \beta \geq 2\left(\xi_{0}-\beta\right)
$$

which is what we need. Q.E.D. for the lemma.

Finally, adding the inequality of Lemma 6.5 to the previous estimates, we obtain an incompatible system of linear equations and inequalities (checked using MAPLE), which completes the exclusion of the case under consideration.

Therefore, the only remaining possibility is when $B^{*}=\Theta \cap Q_{P}$, where $\Theta \subset E_{P}$ is a linear subspace of codimension two. The claim of Lemma 6.4 is valid. Let $R \in$ $\left|H_{P}-\Theta\right|$ be a general divisor, $Z_{R}=\left(Z_{P} \circ R\right)$ an effective cycle of codimension two on $R, Z_{P}=Z_{0}+Z_{1}$ is, as usual, the $T_{P}$-decomposition of the cycle $Z_{P}$. We get the standard set of linear equalities and inequalities for that decomposition: $(24,25,27$, $46,48)$ and (47) with $d_{B}=4$. Now let us consider the cycle $Z_{R}$ more carefully. Set

$$
\left(Z_{i} \circ R\right)=Z_{i}^{\sharp}+c_{i} n^{2} \Delta,
$$

where $\Delta=\mathrm{Bs}\left|H_{P}-\Theta\right|$ is a hyperplane section of the hypersurface $T_{R}$; note that none of the components of the cycle $Z_{1}^{\sharp}$ is not contained in $T_{R}$. The support of the cycle $Z_{2}^{\sharp}$ is contained in $T_{R}$. For the subvariety $\Delta$ we obviously have: $\operatorname{deg} \Delta=M$, mult $_{o} \Delta=2$ and mult $_{B^{*}} \Delta^{+}=1$. Obviously,

$$
\operatorname{mult}_{o}\left(Z_{1} \circ R\right)=\operatorname{mult}_{o} Z_{1}, \quad \operatorname{mult}_{B^{*}}\left(Z_{1} \circ R\right)^{+}=\operatorname{mult}_{B^{*}} Z_{1}^{+} .
$$

Setting $\mu_{i}^{\sharp}=\frac{1}{n^{2}}$ mult $_{o} Z_{i}^{\sharp}$ for $i=0,1$, we obtain the inequality

$$
\mu_{1}^{\sharp}+\left(\xi_{1}-c_{1}\right) \leq \frac{2 M}{M-2}\left(d_{1}-c_{1}\right) .
$$


Furthermore, the following equalities

$$
\operatorname{mult}_{o}\left(Z_{0} \circ R\right)=\operatorname{mult}_{o} Z_{0}, \quad \operatorname{mult}_{B^{*}}\left(Z_{0} \circ R\right)^{+}=\operatorname{mult}_{B^{*}} Z_{0}^{+}
$$

hold. The cycle $Z_{0}^{\sharp}$ is an effective divisor on $T_{R}$, which does not contain $\Delta$ as a component. Let $R^{*} \in\left|H_{P}-\Theta\right|$ be another general divisor. Obviously,

$$
R^{*} \cap T_{R}=R \cap R^{*} \cap T_{P}=\Delta
$$

so that none of the components of the cycle $Z_{0}^{\sharp}$ is not contained in $R^{*}$ and therefore the cycle $Z_{0}^{*}=\left(Z_{0}^{\sharp} \circ R^{*}\right)$ of codimension two on $T_{R}$ is well defined. The cycle $Z_{0}^{*}$ is an effective divisor on $\Delta$. Setting $\mu_{0}^{*}=$ mult $_{o} Z_{0}^{*}$, we obtain the inequality

$$
\mu_{0}^{*} \geq \mu_{0}^{\sharp}+2\left(\xi_{0}-c_{0}\right)
$$

By the regularity conditions on the hypersurface $R$ the inequality

$$
\mu_{0}^{*} \leq \frac{4 M}{M-2}\left(d_{0}-c_{0}\right)
$$

holds. But it is not hard to obtain a stronger estimate. By the regularity conditions and the Lefschetz theorem we have:

$$
\left(\Delta \circ T_{o}\left(T_{R}\right)\right)=\Delta \cap T_{o}\left(T_{R}\right)=\Delta \cap T_{o}\left(T_{P}\right)
$$

is an irreducible reduced divisor on $\Delta$, which has the degree $2 M$ and the multiplicity precisely 6 at the point $o$. Let $Y$ be an irreducible component of the cycle $Z_{0}^{*}$. Then either the inequality $\left(\right.$ mult $\left._{o} / \operatorname{deg}\right) Y \leq(3 / M)$ holds, or $Y$ is not contained in the hypertangent divisor $T_{o}\left(T_{P}\right)$, so that the estimate

$$
\frac{\text { mult }_{o}}{\operatorname{deg}} Y \leq \frac{10}{3(M-2)}
$$

is true. From this it follows, that the inequality

$$
\mu_{0}^{*} \leq \max \left(3, \frac{10 M}{3(M-2)}\right)\left(d_{0}-c_{0}\right)
$$

holds.

Using MAPLE, it is easy to check that the system of linear equations and inequalities for $\mu_{*}, d_{*}, c_{*}, \mu_{*}^{\#}$ and $\mu_{0}^{*}$, obtained above, has no solutions.

The case 2.2 is completely excluded. 


\subsection{Exclusion of the case 2.3}

Assume that the case 2.3 takes place. We have $\mu_{B}=\operatorname{mult}_{B} Z_{P}^{+}>4 n^{2}$, so that we get the following sequence of inequalities:

$$
12 n^{2} \geq \operatorname{mult}_{o} Z_{P} \geq d_{B} \mu_{B}>4 d_{B} n^{2}
$$

where $d_{B}=\operatorname{deg} B \geq 2$ (the case of a linear subspace was excluded by Proposition 5.1), whence we conclude that $d_{B}=2$, that is, $B$ is a quadric in some hyperplane $\Pi \subset E_{P}$.

If $B \not \subset Q_{P}$, then we argue as in Sect. 5.3: we write down $Z_{P}=Z_{0}+Z_{1}$ and intersect $Z_{1}$ with a general divisor $R \in\left|H_{P}-\Pi\right|$. Since $\mu_{B}>4 n^{2}$, we obtain the linear inequalities

$$
\begin{aligned}
& \text { mult }_{o} Z_{0} \leq \frac{3}{M} \operatorname{deg} Z_{0} \\
& \text { mult }_{o} Z_{1}+8 n^{2}<\frac{8}{3(M-2)} \operatorname{deg} Z_{1} \leq \frac{4}{M} \operatorname{deg} Z_{1}
\end{aligned}
$$

which hold for $M \geq 6$. Putting together and recalling that mult $Z_{P}>8 n^{2}$, we obtain a contradiction, excluding the possibility $B \not \subset Q_{P}$.

So let us assume that $B=\Pi \cap Q_{P}$ is a hyperplane section of the quadric $Q_{P}$. Consider $\Delta=\mathrm{Bs}\left|H_{P}-\Pi\right|$, which is a hyperplane section of the variety $T_{P}$. Write down

$$
Z_{P}=a \Delta+Z_{*}
$$

where $a \in \mathbb{Z}_{+}$and $Z_{*}$ does not contain $\Delta$ as a component. For $\Delta$ we have: $\operatorname{deg} \Delta=M$, mult $_{o} \Delta=2$ and $\operatorname{mult}_{B} \Delta^{+}=1$. Therefore, $\operatorname{deg} Z_{*}=\left(4 n^{2}-a\right) M$, and for the multiplicities we have the equalities

$$
\operatorname{mult}_{o} Z_{*}=\mu-2 a, \quad \operatorname{mult}_{B} Z_{*}^{+}=\mu_{B}-a .
$$

Now, arguing in word for word the same way as above in the case $B \not \subset Q_{P}$, where $Z_{P}$ is replaced by $Z_{*}$, we obtain a contradiction. The case 2.3 is excluded.

Proof of Theorem 1.5 is complete.

Open Access This article is distributed under the terms of the Creative Commons Attribution 4.0 International License (http://creativecommons.org/licenses/by/4.0/), which permits unrestricted use, distribution, and reproduction in any medium, provided you give appropriate credit to the original author(s) and the source, provide a link to the Creative Commons license, and indicate if changes were made.

\section{References}

1. Call, F., Lyubeznik, G.: A simple proof of Grothendieck's theorem on the parafactoriality of local rings. Contemp. Math. 159, 15-18 (1994)

2. Clemens, H.: Degeneration techniques in the study of threefolds. Lect. Notes Math. 947, 93-154 
3. Clemens, H., Griffiths, Ph: The intermediate Jacobian of the cubic threefold. Ann. Math. 95, 281-356 (1972)

4. Cheltsov, I.A.: Local inequalities and the birational superrigidity of Fano varieties. Izvestiya: Mathematics 70(3), 605-639 (2006)

5. Eckl, Th., Pukhlikov, A.: On the locus of non-rigid hypersurfaces. In: Automorphisms in Birational and Affine Geometry. Springer Proceedings in Mathematics and Statistics, vol. 79, pp. 121-139 (2014)

6. Fano, G.: Nuove ricerche sulle varieta algebriche a tre dimensioni a curve-sezioni canoniche. Comm. Rent. Ac. Sci. 11, 635-720 (1947)

7. de Fernex, T.: Fano hypersurfaces and their birational geometry. In: Automorphisms in Birational and Affine Geometry. Springer Proceedings in Mathematics and Statistics, vol. 79, pp. 103-120. Springer, Berlin (2014)

8. Fulton, W.: Intersection Theory. Springer, Berlin (1984)

9. Graber, T., Harris, J., Starr, J.: Families of rationally connected varieties. J. Am. Math. Soc. 16(1), 57-67 (2002)

10. Grinenko, M.M.: On the double cone over the Veronese surface. Izvestiya: Mathematics 67(3), 421-438 (2003)

11. Grinenko, M.M.: Mori structures on Fano threefold of index 2 and degree 1. Proc. Steklov Inst. Math. 246(3), 103-128 (2004)

12. Holme, A.: Codimension 2 subvarieties of projective space. Manuscr. Math. 65, 427-446 (1989)

13. Iskovskikh, V.A., Manin, Yu.I.: Three-dimensional quartics and counterexamples to the Lüroth problem. Math. USSR Sb. 86(1), 140-166 (1971)

14. Kollár, J., et al.: Flips and abundance for algebraic threefolds, vol. 211. Asterisque-Societe Mathematique de France, Paris (1992)

15. Kollár, J.: Nonrational hypersurfaces. J. Am. Math. Soc. 8, 241-249 (1995)

16. Kollár, J.: Nonrational covers of $C P^{m} \times C P^{n}$. In: Explicit Birational Geometry of Threefolds. London Mathematical Society Lecture Note Series, vol. 281, pp. 51-71. Cambridge University Press, Cambridge (2000)

17. Pukhlikov, A.V.: Birational automorphisms of Fano hypersurfaces. Invent. Math. 134(2), 401-426 (1998)

18. Pukhlikov, A.V.: Birationally rigid Fano complete intersections. Crelle J. für die reine und Angew. Math. 541, 55-79 (2001)

19. Pukhlikov, A.V.: Birationally rigid Fano hypersurfaces. Izvestiya: Mathematics 66(6), 1243-1269 (2002)

20. Pukhlikov, A.V.: Birational geometry of algebraic varieties with a pencil of Fano complete intersections. Manuscr. Math. 121, 491-526 (2006)

21. Pukhlikov, A.V.: Birationally rigid varieties. I. Fano varieties. Russ. Math. Surv. 62(5), 857-942 (2007)

22. Pukhlikov, A.V.: Birational geometry of singular Fano varieties. Proc. Steklov Inst. Math. 264, 159-177 (2009)

23. Pukhlikov, A.V.: Birational geometry of Fano double spaces of index two. Izvestiya: Mathematics 74(5), 925-991 (2010)

24. Pukhlikov, A.: Birationally Rigid Varieties. Mathematical Surveys and Monographs, vol. 190. AMS, Providence (2013)

25. Totaro, B.: Hypersurfaces that are not stably rational. arXiv: 1502.04040 